\title{
Muscle specific translational control of Cand2 by mTORC1 regulates adverse cardiac remodeling
}

Agnieszka A. Gorska ${ }^{1,2 \#, ~ C l a r a ~ S a n d m a n n ~}{ }^{1,2 \#}$, Eva Riechert ${ }^{1,2}$, Christoph Hofmannn ${ }^{1,2}$, Ellen Malovrh $^{1,2}$, Eshita Varma ${ }^{1,2}$, Vivien Kmietczyk ${ }^{1,2}$, Lonny Jürgensen ${ }^{1,2}$, Verena Kamuf-Schenk ${ }^{1,2}$, Claudia Stroh ${ }^{1,2}$, Jennifer Furkel ${ }^{1,2}$, Matthias H. Konstandin, Carsten Sticht ${ }^{3}$, Etienne Boileau $^{1,2,4}$, Christoph Dieterich ${ }^{1,2,4}$, Hugo A. Katus ${ }^{1,2}$, Shirin Doroudgar ${ }^{1,2}$ and Mirko Völkers ${ }^{\star 1,2}$

${ }^{1}$ Department of Cardiology, Angiology and Pneumology, University Hospital Heidelberg, Im 10 Neuenheimer Feld 410, 69120 Heidelberg, Germany

$11{ }^{2}$ DZHK (German Centre for Cardiovascular Research), partner site Heidelberg/Mannheim, 12 Germany

$13{ }^{3}$ Medical Research Center, Medical Faculty Mannheim, Heidelberg University, Mannheim, 14 Germany

154 Section of Bioinformatics and Systems Cardiology, Department of Cardiology, Angiology, and 16 Pneumology and Klaus Tschira Institute for Integrative Computational Cardiology, University 17 of Heidelberg, Heidelberg, Germany

18 \# authors contributed equally

19

20 Running title: Cand2 and cardiac remodeling

\section{Correspondence:}

Dr. Mirko Völkers

email: mirko.voelkers@med.uni-heidelberg.de

29. Keywords: Cand2/ Cardiac/ Hypertrophy/mTOR 
Abstract

The mechanistic target of rapamycin (mTOR) is a key regulator of pathological remodeling in the heart by activating ribosomal biogenesis and mRNA translation. Inhibition of mTOR in cardiomyocytes is protective, however, a detailed role of mTOR in translational regulation of specific mRNA networks in the diseased heart is largely unknown. A cardiomyocyte genomewide sequencing approach was used to define mTOR-dependent post-transcriptional gene expression control at the level of mRNA translation. This approach identified the musclespecific protein Cullin-associated NEDD8-dissociated protein 2 (Cand2) as a translationally upregulated gene, dependent on the activity of mTOR. Deletion of Cand2 protects the myocardium against pathological remodeling. Mechanistically, we found that Cand2 links mTOR signaling to pathological cell growth by increasing Grk5 protein expression. Our data suggest that cell-type-specific targeting of mTOR might have therapeutic value for adverse pathological cardiac remodeling.

\section{Non-standard Abbreviations and acronyms}

Cand2

CPM

RPKM

DEGs

IP

mTOR

nt

NRCMs

LV

RPF

Ribo-seq

RNA-seq

TOP-motif

5'UTR

IRES

TAC

PE

CX

PLA

WT

$\mathrm{KO}$

$\mathrm{KD}$

$\mathrm{OE}$
Cullin-associated NEDD8-dissociated protein 2

Count Per Million

Reads Per Kilobase Million

Differential Expressed Genes

Immunoprecipitation

Mechanistic Target Of Rapamycin

Nucleotide

Neonatal Rat Cardiac Myocytes

Left ventricle

Ribosome Protected mRNA Fragments

Ribosomal sequencing

RNA sequencing

Terminal oligo pyrimidin motif

5' Untranslated Region

Internal Ribosomal Entry Site

Transverse Aortic Constriction

Phenylephrine

Cycloheximide

Proximity Ligation Assay

Wild-type

Knock-out

Knock-down

Overexpression 
bioRxiv preprint doi: https://doi.org/10.1101/2020.11.29.403196; this version posted December 1, 2020. The copyright holder for this preprint (which was not certified by peer review) is the author/funder. All rights reserved. No reuse allowed without permission.

\section{Introduction}

Pathological cellular remodeling is a hallmark of heart failure (HF) independent from the underlying etiology such as pressure overload, myocardial infarction, or inherited cardiomyopathies. Several studies and our recent work revealed that pathological cardiac stress induces early morphological changes and cardiac hypertrophy due to the activation of the kinase mechanistic target of rapamycin complex 1 (mTORC1)(Buss et al, 2009; Völkers et al, 2013; Sciarretta et al, 2018; Zhang et al, 2010). mTORC1 promotes protein synthesis by induction of rRNA transcription, ribosomal protein synthesis, phosphorylation of translation initiation factors and enhances translation of specific mRNAs that are required for stress adaptation (Ma \& Blenis, 2009).(ladevaia et al, 2012). Mechanistically, mTORC1 promotes translation of a specific subset of mRNAs regulated by the translation initiation factor elF4E by direct phosphorylation of the elF4E binding proteins (4E-BPs) (Thoreen et al, 2012). (Hsieh et al, 2012). mTORC1-sensitive transcripts often contain a terminal oligo-pyrimidine (TOP) or TOP-like motif in the 5' UTR and mTORC1-dependent transcripts are predominantly regulated on the translational level (Jefferies et al, 1994) (Avni et al, 1996),(Thoreen et al, 2012).

Systemic pharmacological or genetic mTORC1 inhibition prevents pathological hypertrophy and improves cardiac function in murine disease models (Buss et al, 2009; Shioi et al, 2003; Völkers et al, 2013), but no established therapeutic regime targets mTORC1 at the level of cardiomyocytes in patients yet. Moreover, the role of mTORC1 in translational regulation of specific mRNA networks in the diseased heart is largely unknown, partly because existing tools in previous studies were unable to analyze gene expression at the level of translation.

We aimed to characterize mTORC1-dependent changes in gene expression that mediate cardiac response to pathological stress. Ribosome profiling (Ribo-seq) was used to obtain quantitative measurements of translation, and to dissect the mTORC1-dependent translational regulation of gene expression in cardiomyocytes. Among other genes dependent on the activity of mTORC1 was the uncharacterized muscle-specific gene Cullin-associated NEDD8-dissociated protein 2 (Cand2). Initially named transcription factor TATA-binding protein 120B (TIP120B), Cand2 belongs to the TIP120 gene family and shares $60 \%$ homology with TIP120A (Cand1) (Aoki et al, 1999).

In contrast to ubiquitously expressed Cand1, Cand2 is a muscle-specific protein and has been identified only in mammals. Both proteins have been shown to directly bind and modulate the activity status of Cullin1 (Cul1) (Liu et al, 2002)'(Goldenberg et al, 2004)'(Shiraishi et al, 2007). Cul1 is a component of SCF-like E3 ligase complex that promotes ubiquitination (Zheng et al, 2002), (Furukawa et al, 2000), (Zou et al, 2018). The activation of the SCF complex is stimulated by reversible and covalent post-translational modification (neddylation) of CUL1, which relies on ligation of the ubiquitin-like polypeptide Nedd8 protein to a specific lysine residue in the C-terminus (Goldenberg et al, 2004),(Min et al, 2005).

We found that pressure overload induced Cand2 expression on the level of translation which depends on the activity of mTORC1. Cand2 is required and sufficient for pathological growth. Cand2 knock-out mice are protected against pathological remodeling. Mechanistically, Cand2 post-transcriptionally controls the expression of G-protein coupled receptor 5 (Grk5) expression in vitro and in vivo, which in turn is linked to transcription of hypertrophic genes driven by myocyte enhancer factor 2 (MEF2).

Our data highlighted a novel mechanism where translational, mTORC1-dependent, control of Cand2 expression results in increased expression of Grk5 which results in transcriptional reprogramming in diseased cardiac myocytes (mRNA translation controls transcriptional activity). This linked mTOR dependent cytosolic signaling events, that drive specific mRNA translation, to transcriptional activity. 
bioRxiv preprint doi: https://doi.org/10.1101/2020.11.29.403196; this version posted December 1, 2020. The copyright holder for this preprint (which was not certified by peer review) is the author/funder. All rights reserved. No reuse allowed without permission.

\section{Results Cand2 is a myocyte-specific protein translationally upregulated during pathological stress}

To define mRNAs translationally regulated by mTOR signaling in response to pathological cell growth, cultures of neonatal rat ventricular cardiomyocytes (NRCMs) were acutely treated with the $\alpha-1$ adrenoreceptor agonist phenylephrine (PE) and with Torin 1 , which inhibits mTOR by binding to the ATP-binding site in the kinase domain (Thoreen et al, 2012). Specific inhibition of mTOR-dependent protein signaling by Torin 1 was confirmed by immunoblots (EV1). Ribosome profiling (Ribo-seq) has emerged as a quantitative technique to study global gene expression, and overcomes limitations of classical expression analysis, as it directly quantifies the number of translating ribosomes (Ingolia et al, 2009). The effect of Torin 1 on mRNA translation was analyzed by Ribo-seq in cultured cardiomyocytes (Fig. 1A). Total cellular RNA was also collected for parallel RNA-seq to quantify mRNA abundance (the full list is provided in Source Data set).

We identified 199 genes that decreased and 165 that increased $\left(\log _{2} F C>1\right.$ or $\left.<-1\right)$ in Ribo-seq in an mTOR-dependent manner in response to PE treatment (Fig. 1B). Among the suppressed genes, $17 \%$ have a known TOP or TOP-like motif in the 5' UTR (Fig. 1C), which is known to be sensitive to mTOR inhibition (Thoreen et al, 2012), and the suppressed genes are predominantly regulated on the translational level (Fig. 1D). Immunoblotting confirmed a decrease in the expression of selected mTOR-dependent genes such as eEF2 and rpS5 at the protein level upon Torin 1 treatment (EV1) but not at the transcript level. In contrast, molecular markers of pathological growth such as Nppb and Nppa were still induced (EV1). Stimulation of NRCMs with PE resulted in a strong increase in mRNAs in polysomal fractions (EV1). The increase of mRNAs in the polysomal fraction induced by PE was blocked by Torin 1 , confirming that Torin 1 blocks the recruitment of ribosomes into polysomal fractions. The effects of inhibiting of mTOR on cardiac myocyte growth were examined in isolated cardiac myocytes stimulated with PE for 24h. Torin 1 inhibited PE-induced hypertrophy as assessed by cell surface area measurements (EV2). To measure the effect of Torin 1 on cap-dependent translation in cells, we used a dual-luciferase reporter vector that distinguishes cap-dependent versus cap-independent translation by separating Renilla luciferase from firefly with the Polio IRES(Poulin et al, 1998) (EV2). Therefore, the Renilla/firefly ratio would determine the capdependent translation ratio. Torin 1 inhibited cap-dependent, but not IRES-dependent translation measured by luciferase activity.

In line with the in vitro data, mTOR inhibition with Torin 1, in vivo, blocked pathologic growth induced by acute transverse constriction (TAC) surgery, a common model for cardiac hypertrophy and remodeling (EV2) (Doroudgar et al, 2019). Thus, mTOR-dependent and capdependent protein synthesis are increased during hypertrophic growth and this is necessary for induction of cell growth, in vitro and in vivo.

Gene ontology (biological processes) analysis of the genes that were suppressed by Torin 1 showed strong enrichment for those involved in translation, metabolism, as well as signaling cascades (Fig. 1E). Overall, this dataset defined a specific subset of genes that are regulated in an mTOR-dependent manner in response to neurohumoral stimulation with PE. Next, we followed our identified mTOR-dependent genes in a previously published in vivo data set after TAC surgery (Doroudgar et al, 2019). mTOR-dependent genes were translationally upregulated in response to TAC surgery (Fig. 1F-G). Increased expression of identified mTOR targets independent from changes of transcript levels such as eEF2, Desmin, rpS5, or rpS20 were confirmed using immunoblots and RT-qPCRs from heart lysates after TAC surgery (Fig. 1H-I).

Among the 199 genes with decreased translation upon Torin 1 treatment, 17 were significantly increased in the in vivo data set after TAC surgery (Doroudgar et al, 2019) (Fig. 2A). Among this subset of genes that were increased by pressure overload in vivo and dependent on mTOR activity in vitro, was Cand2, a muscle specific protein with unknown role in the heart ${ }^{12}$. (Table Fig. 2A). Since its role in cardiomyocytes was completely unknown, we aimed to characterize the role of Cand2 during pathological remodeling. Torin 1-induced 
bioRxiv preprint doi: https://doi.org/10.1101/2020.11.29.403196; this version posted December 1, 2020. The copyright holder for this preprint (which was not certified by peer review) is the author/funder. All rights reserved. No reuse allowed without permission.

downregulation of Cand2 translation was not due to transcriptional regulation assessed by parallel RNA-seq (Fig. 2B). mTOR activity increased Cand2 protein levels in vitro, as well, but had no impact on its transcript levels measured by RT-qPCR (Fig. 2C-D), confirming the results from the Ribo-seq data sets. Ribo-seq data revealed an increased expression of Cand2 two days after TAC (Fig. 2E). In contrast, Cand2 translation was unchanged in early (3 hours after TAC) and chronic pressure overload (2 weeks after TAC) (Fig. 2F-G). Translational upregulation of Cand2 in the heart was also detected on the protein level when compared by immunoblotting to sham-operated mice (Fig. 2H). Increased Cand2 levels in vivo were also not caused by increased Cand2 transcript levels as shown by RT-qPCR (Fig. 2I). To assess whether Cand2 expression is controlled by mTORC1 in vivo, Torin 1 was injected in mice after TAC or sham surgery and Cand2 levels were assessed by immunoblotting (Fig. 2H). The known mTORC1-sensitive 40S ribosomal protein 5 (rpS5) was used as a positive control (Uprety et al, 2018). Along with rpS5 inhibition by Torin 1, increased Cand2 protein levels after TAC surgery were completely blocked by Torin 1, suggesting that Cand 2 mRNA belongs to mTORC1-sensitive genes, in vivo, as well. Since mTORC1 specifically regulates translation of mRNAs with 5'TOP motifs, we placed the 5' UTR of Cand2 mRNA upstream of Renilla luciferase to investigate its regulatory potential in a mTORC1-dependent manner (Fig. 2J). Cand2 5' UTR showed a similar degree of reporter inhibition with Torin 1 treatment as the known TOP motif-containing 5' UTR of eEF2. This confirmed mTORC1-dependent translation of Cand2 and suggests the presence of a regulatory motif in its 5' UTR. Taken together, mTORC1 controls the expression levels of Cand2 in response to pathological stimulation both in vitro and in vivo.

To further characterize Cand2, we analyzed its expression profile in different organs in mice. Consistently with a previous report (Aoki et al, 1999), Cand2 protein was expressed predominantly in muscle tissues and was highly abundant in the left ventricle (Fig. 3A). Quantitative RT-PCR analysis also revealed the highest Cand2 mRNA level in the heart (Fig. 3B). Immunofluorescent staining confirmed specific Cand2 expression only in cardiomyocytes (Fig. 3C), suggesting that Cand2 protein expression is restricted to muscle cells in the heart. Subsequently, we analyzed Cand2 subcellular localization in isolated cardiomyocytes by immunofluorescent staining (Fig. 3D). Consistently with studies on skeletal muscle cell maturation (Shiraishi et al, 2007), endogenous Cand2 was located in the nucleus and cytoplasm in cardiac myocytes. Subcellular fractionation of the left ventricle confirmed the cytoplasmic and nuclear distribution of Cand2 (Fig. 3E).

\section{Cand2 contributes to cardiomyocytes pathological growth in vitro and in vivo}

Next, we studied the effect of Cand2 on cardiomyocyte cell size after Cand2 knockdown or overexpression (Fig. 4A-B). Cand2 depletion in vitro decreased cell surface area and blunted the response to neurohormonal stimulation with PE treatment (Fig. 4C-D). In contrast, Cand2 overexpression induced cardiomyocyte growth, and the increase in cell size after overexpression was comparable to control cells after PE (Fig. 4E). Nppa levels correlated to Cand2 expression: they were decreased when Cand2 was depleted and elevated in Cand2 overexpression in untreated and PE stimulated cardiomyocytes (Fig. 4F).

To study the role of Cand2 in vivo, a novel Cand2 knock-out (KO) mouse was generated (EV3). Cand2 deficient mice were viable and cardiac function as assessed by ejection fraction, as well as left ventricle weight to body weight ratio remained unchanged compared to littermate control animals (EV3). In line with this, Cand2 KO did not alter the levels of fetal gene markers normally upregulated in the adult heart during stress, such as Nppa and Nppb (EV3). Moreover, Cand2 knock-out did not disturb the levels of Cand1 (EV3)

Next, Cand2 KO mice along with wild-type (WT) littermates were subjected to TAC or sham surgery and phenotypic consequences were analyzed 4 weeks after surgery. TAC operation resulted in decreased ejection fraction, fractional shortening and increased LV/BW ratio in WT mice (Fig. 4G-I). In contrast, Cand2 deficient mice showed preserved cardiac function as well as significantly decreased LV/BW ratio compared to TAC-operated WT mice. Moreover, TAC-induced Nppa and Nppb levels in WT mice were 1.9-fold and 3-fold reduced 
bioRxiv preprint doi: https://doi.org/10.1101/2020.11.29.403196; this version posted December 1, 2020. The copyright holder for this preprint (which was not certified by peer review) is the author/funder. All rights reserved. No reuse allowed without permission.

in Cand2 KO mice, respectively (Fig. 4J). Overall, these results suggest that Cand2 deletion protects against pathological remodeling in response to pressure overload.

\section{Cand2 controls Grk5 expression and affects MEF2-dependent transcription}

Next, we performed transcriptome profiling of left ventricles from wild-type and Cand2 knock-out mice to identify targets that might be regulated by Cand2. mRNA sequencing identified only small changes in the transcriptome when Cand2 was deleted (Fig. 5A). Interestingly, pathway analysis revealed that most of the Cand2-dependent genes are enriched in categories regulating transcriptional control and proteasomal function. Among the Cand2dependent group of genes involved in transcriptional control, the G-protein coupled receptor kinase 5 (Grk5) has been one of the most regulated genes in Cand2 knock-out mice (Fig. 5A). While the canonical role of Grks is to phosphorylate agonist bound G-protein coupled receptors, which promotes the binding of an arrestin protein to the receptor, resulting in subsequent desensitization of the receptor, Grk5 has been found to localize to the nucleus via a nuclear localization sequence. Previous work showed that following pressure overload, Grk5 accumulates in the nucleus of cardiomyocytes and acts as a class II histone deacetylase (HDAC) kinase, phosphorylating HDAC5 specifically, leading to its nuclear export and derepression of the transcription factor MEF2 (Zhang et al, 2011).

First, we analyzed Grk5 expression after gain- or loss-of-function of Cand2 in vitro in neonatal rat ventricular myocytes (NRCMs) (Fig. 5B-C). Cand2 overexpression increased Grk5 protein in NRCMs but did not change its mRNA levels. In contrast, Grk5 protein was downregulated in Cand2 knock-down and Grk5 transcript level again remained unaffected. Next, we further analyzed levels of Grk5 in Cand2 KO mice in TAC-induced pressure overload 2 weeks after surgery (Fig. 5D). Quantitative RT-PCR analysis did not reveal any significant changes in Grk5 mRNA levels after TAC in Cand2 KO mice (Fig. 5D). In line with other reports, Grk5 protein was highly increased in TAC-operated wild-type mice. Lack of Cand2 led to an approximately 10-fold decrease of Grk5 protein levels after TAC. Since Grk5 has been shown to translocate to the nucleus of cardiomyocytes following pressure overload via its nuclear localization signal (NLS) (Johnson et al, 2004),(Martini et al, 2008)'(Gold et al, 2013), we examined Grk5 levels in the cytoplasm and nucleus in vivo in Cand2 KO mice (Fig. 5E). Neither TAC nor lack of Cand2 affected Grk5 levels in the cytoplasmic fraction, but Grk5 levels increased in the nucleus in WT mice in response to TAC, which was blocked in Cand2 mice.

Since Grk5 regulates the activity of the transcription factor MEF2, we analyzed MEF2 activity by using a luciferase reporter assay. Cand2 knock-down decreased MEF2 luciferase activity by 3.5-fold in vitro (Fig. 6A). Moreover, the expression of direct MEF2-dependent mRNAs such as Nr4a1 and Xirp2 (Lehmann et al, 2018),(Huang et al, 2006) were downregulated when Cand2 was depleted (Fig. 6B). Conversely, the levels of Nr4a1 and Xirp2 increased significantly in NRCMs overexpressing Cand2 (Fig. 6C). Inline, levels of Xirp2 increased in WT mice in response to TAC but were blocked in Cand2 KO mice (Fig. 6D). Similarly, mTOR inhibition with Torin1 resulted in decreased Grk5 protein levels and MEF2 luciferase activity in NRCMs, without changes in mRNA levels of Grk5 (Fig. 6E). Next, we confirmed decreased Cand2 and Grk5 protein but not transcript levels after depletion of Raptor, the essential component of mTORC1, in myocytes (Fig. 6F). Raptor knockdown caused decreased protein levels of Cand2 and Grk5 (Fig. 6G). Moreover, RT-qPCR analysis revealed downregulation of direct MEF2 targets such as Nr4a1 and Xirp2 as well as Nppa after knockdown of Raptor (Fig. 6H), suggesting that Grk5 expression and Grk5-mediated MEF2 activity are under direct mTORC1 control. Overall, these results suggest that Cand2 dependent regulation of Grk5 levels is associated with increased MEF2 transcriptional activity and similarly to Cand2, Grk5-MEF2 is downstream of mTORC1 as well.

\section{Cand2 regulates Grk5 protein level by Cullin1 neddylation inhibition.}

Our findings showed that Cand2 depletion results in decreased Grk5 protein levels independent from changes in transcript levels (Fig. 5B), suggesting post-transcriptional regulation of Grk5 expression by Cand2. Cand proteins modulate the activity of Cullin-RING E3 ubiquitin ligases (CRLs). The activity of CRLs is largely dependent on Cullin neddylation, 
bioRxiv preprint doi: https://doi.org/10.1101/2020.11.29.403196; this version posted December 1, 2020. The copyright holder for this preprint (which was not certified by peer review) is the author/funder. All rights reserved. No reuse allowed without permission.

whereby a covalent modification of Cullin1 with the ubiquitin-like protein Nedd8 will induce the conformational rearrangement of Cullin1. Cand2 protein has been shown to interact with and sequester unneddylated Cullin1. To find out whether Cand2, Cullin1, and Grk5 directly interact in cardiomyocytes, co-immunoprecipitations were performed. Endogenous Cullin1 as well as Grk5 was detected after Cand2 immunoprecipitation, whereas Cul4 did not bind Cand2 (EV4). Additionally, we performed proximity ligation assay (PLA) in neonatal cardiomyocytes isolated from wild type and Cand2 knock-out mice (EV4). PLA signal was detected in cardiomyocytes expressing endogenous Cand2, but not in $\mathrm{KO}$ cells, suggesting that Cand2 is associated with Grk5 specifically. Although both proteins were observed ubiquitously in the cell, PLA signal was concentrated in the nucleus. These results support the idea that Cand2 interacts in a complex with Cullin1 with Grk5.

Cand proteins selectively bind to the unnedyladed pool of Cullin1, thus affecting SCF ubiquitin ligase complex (Liu et al, 2002). Thus, we sought to determine the effect of Cand2 on the overall neddylation status of Cullin1 in cardiomyocytes overexpressing and depleted of Cand2 (Fig. 7A and B). Neddylated Cullin1 migrates slower during electrophoresis and can, therefore, be distinguished from unnedylated Cul1. Manipulation of Cand2 expression affected only the neddylated Cul1 levels, whereas the unnedylated fraction remained unchanged. The amount of neddylated Cul1 was slightly but significantly increased when Cand2 was silenced and reduced in Cand2 overexpressing cells, suggesting that Cand2 may regulate Cul1 activity (Fig. 7A-B).

In line with previous reports (Zou et al, 2019), inhibition of neddylation by using a specific inhibitor (MLN4924) was sufficient to induce cardiomyocyte hypertrophy, which was completely blocked by Cand2 knock-down (Fig. 7C). Interestingly, we found that MLN4924 mediated inhibition of Cul1 neddylation alone was sufficient to induce an approximately 2.5fold increase of Grk5 protein levels, independent from transcript levels measured by RT-qPCR (Fig. 7D). The deneddylation of Cul1 by MLN4924 led to approximately 2.5 -fold increase of Grk5 protein, independently from transcript levels measured by RT-qPCR. This induction was largely attenuated after Cand2 knock-down, indicating that Cand2 is required for the induction of Grk5 after neddylation inhibition (Fig. 7D). Additionally, neddylation inhibition did not affect Cand2 transcript levels (EV5). Neither Cand2 knock-down nor overexpression influenced Cul1 mRNA levels, indicating that Cand2 interacts with Cul1 on the protein level (EV5). To show that Cand2 regulates Grk5 protein stability, we assessed Grk5 half-life in cycloheximide chase assay (Fig. 7E). The Grk5 protein half-life was prolonged from approximately 18 to 27 hours when Cand2 was overexpressed suggesting that Grk5 is stabilized by Cand2.

Similar to neddylation inhibition, knock-down of Cul1 increased Grk5 protein amount by 2-folds (Fig. 7F). This strongly suggests that Grk5 degradation is mediated by Cullin-RING E3 ubiquitin ligase complex. Quantitative RT-PCR analysis revealed that all changes in Grk5 levels caused by MLN4924 treatment or Cand2/Cul1 silencing resulted independent from transcriptional changes (Fig. 7D-F).

Finally, to confirm that Cand2 affects cell size through Grk5, we examined whether Grk5 re-expression is sufficient to rescue the decreased cell size phenotype of Cand2-deficient cardiomyocytes. Grk5 overexpression was confirmed by immunoblot and was also functional in Cand2 knock-out cells (Fig. 7G). While Cand2 knock-down again attenuated the hypertrophic growth response of cardiomyocytes, Grk5 overexpression was sufficient to restore hypertrophic cell growth to similar levels of control cardiomyocytes treated with PE. Taken together, we suggest a novel myocyte-specific and mTORC1-mediated signaling cascade. mTORC1-dependent, control of Cand2 expression results in activation of the prohypertrophic Grk5-HDAC4-MEF2 axis via post-transcriptional regulation of Grk5 protein levels by the Cul1-SCF ubiquitin ligase complex (Fig. 7H). 
bioRxiv preprint doi: https://doi.org/10.1101/2020.11.29.403196; this version posted December 1, 2020. The copyright holder for this preprint (which was not certified by peer review) is the author/funder. All rights reserved. No reuse allowed without permission.

\section{Discussion}

This study provides evidence for a novel role of Cand2, a component of the SCF (SKP1Cul1-F-box protein) E3 ubiquitin ligase complex, in the myocardium. Cand2 is a musclespecific protein that we identified in a cardiomyocyte genome-wide screen for mTORC1dependent gene expression control at the level of mRNA translation. Several studies and our own work showed that mTORC1-signaling controls physiological as well as pathological myocardial remodeling (Sciarretta et al, 2018; Völkers et al, 2014, 2013). The central role of mTORC1 in integrating multiple intra- and extracellular parameters requires an elaborate control system to accomplish fine-tuning of signaling to downstream targets. Clinically, targeting mTORC1 with rapamycin is already an established application to prevent re-stenosis after percutaneous coronary stent implantation. Systemic pharmacological or genetic mTORC1 inhibition prevented pathological hypertrophy and improved cardiac function in murine disease models (Buss et al, 2009; Shioi et al, 2003; Völkers et al, 2013). Still, the identity of $\mathrm{mTORC} 1$ translationally regulated mRNAs in the diseased heart were largely unknown.

Cand2 is a translationally upregulated gene dependent on the activity of mTORC1 during pathological stress both in vitro and in vivo, whereas Cand2 expression is not regulated during physiological stress. We found that Cand2 is required for pathological hypertrophy in cardiomyocytes by upregulation of the kinase Grk5 which is associated with the induction of pathological gene expression profiles. Indeed, Cand2 knock-out mice were protected against pathological remodeling, and cardiac dysfunction, which was associated with reduced levels of Grk5 and MEF2-dependent gene expression profiles. Conversely, overexpression of Cand2 was sufficient to drive pathological hypertrophy and gene expression in vitro. Cand2 expression was also found to be upregulated in human cardiomyopathy patients (van Heesch et al, 2019).

Cand2 was initially identified as a muscle-specific homolog of Cand1 and its only described function was the acceleration of myogenesis in skeletal myoblasts during differentiation (Aoki et al, 1999; Shiraishi et al, 2007). Interestingly, Cand2 has been linked to atrial fibrillation susceptibility using expression quantitative trait loci mapping, suggesting that it might play an important role in atrial myocytes as well. Increased levels of Cand2 correlated with a higher incidence of atrial fibrillation (Sinner et al, 2014; Wei et al, 2016; Gregers et al, 2017), suggesting that Cand2 might drive pathological remodeling in the atrium.

Mechanistically, Cand proteins modulate the activity of Cullin-RING E3 ubiquitin ligases (CRLs). The substrate-binding domain of Cullins, through their adaptors, can recruit hundreds of known substrate receptors that specifically target an even larger array of substrates. It has become increasingly evident that the cardiac ubiquitin-proteasomal function is not only highly dynamic but is also critical for healthy myocardium (Drews \& Taegtmeyer, 2014). Just as CRLs regulate protein levels and function by covalent modification, they are subjected to modifications at the posttranslational level, which in turn regulate ligase activity. NEDD8, a ubiquitin-like protein, is covalently attached to a conserved lysine residue in the C-terminal Cullin homology domain. The activity of CRLs is largely dependent on Cullin neddylation, whereby Nedd8 induces conformational rearrangement of Cullins. Cand proteins have been shown to interact with and sequester unneddylated Cullins (Liu et al, 2018). Thus, Cand proteins have been initially characterized as inhibitors of Nedd8 conjugation, which initially implied that Cand proteins are negative regulators of CRLs. However, subsequent studies of Cand1 deficient cells and organisms suggested also a positive regulatory function of Cand on CRLs (Liu et al, 2018). Importantly, neddylation homeostasis is crucial for the integrity of the heart. Complete loss of neddylation in cardiac myocytes resulted in dilated cardiomyopathy with premature death (Su et al, 2013, 2011). Pharmacological inhibition of neddylation using a selective neddylation inhibitor MLN4924 promoted pathological hypertrophy and resulted in cardiac failure (Zou et al, 2018, 2019).

Neddylation of Cullins can provide a regulatory mechanism that fine-tunes substrate ubiquitination and targeting for proteasomal degradation. We found that Cand2 regulates 
protein levels of Grk5 predominantly independent of the transcript level. Previous work showed that following pressure overload, Grk5 accumulated in the nucleus of cardiomyocytes and acts in the nucleus as a class II histone deacetylase (HDAC) kinase, phosphorylating specifically HDAC5 leading to its nuclear export and de-repression of the transcription factor MEF2 as well as NFAT (Cannavo et al, 2016; Traynham et al, 2016). Furthermore, transgenic mice with Grk5 cardiac overexpression could not cope with pressure overload (Martini et al, 2008). Once free of repression, MEF2 and NFAT are responsible for the transcription of hypertrophic genes which leads to pathological hypertrophy (Zhang et al, 2011).

Here we found that reduced levels of Grk5 in Cand2 depleted myocytes resulted in decreased MEF2 activity. We also confirmed the direct interaction of Cand2 with Cullin1 and Grk5 in myocytes, supporting the hypothesis that Cand2 controls Cullin1-dependent Gr5k levels in cardiomyocytes. Moreover, pharmacological inhibition of neddylation using a selective neddylation inhibitor MLN4924 resulted in loss of Cullin1 neddylation and increased cell size, which was associated with increased Grk5 levels independent from transcript levels. Cardiomyocytes depleted of Cand2 did not respond to MLN4924, suggesting that Cand2 is necessary for growth induction caused by MLN4924. Rescue of Grk5 expression in Cand2 depleted cells reversed the observed phenotype with increased pathological cell size similar to control cells.

Taken together, we suggest a novel mechanism by which pathological mTORC1 signaling links translationally-controlled Cand2 expression to transcriptional activity (mRNA translation controls transcriptional activity). We suggest that these findings might be transferable toward novel treatment options. We hypothesize that Cand2 is a promising target as it is a cardiac muscle-specific protein, early (translationally) upregulated during disease initiation, and sufficient for pathological remodeling. Future studies will develop a nucleic acid-based therapy against Cand2. Recent advances in nucleic acid-based therapeutics show promising results in treatments for various diseases including pathological cardiac hypertrophy and it should be possible to target Cand2 translation by antisense oligonucleotides targeting the 5' UTR of Cand2 (Morihara et al, 2017). Future studies are also needed to identify additional interaction partners of Cand2 dependent on the neddylation status of Cullin1 to understand how mTORC1-dependent upregulation of Cand2 affects the stability of protein networks in the nucleus.

\section{Material and Methods}

The authors declare that all supporting data are available within the article (and in the Online Data Supplement).

Raw sequencing data have been made publicly available and can be accessed. RNA-Seq: Raw data have been uploaded to GEO (accession ID: GSE153364)

Ribo-Seq: Raw data have been uploaded to SRA (accession ID:SRP156230)

\section{Parallel Generation of Ribo-seq and RNA-seq Libraries}

Ribo-seq and RNA-seq libraries were prepared for each biological replicate. Ribosome footprints were generated after immunoprecipitation of cardiac myocyte-specific monosomes with anti-HA magnetic beads after treating the lysate with RNase I. Libraries were generated according to the mammalian Ribo-seq kit (Illumina). Barcodes were used to perform multiplex sequencing and create sequencing pools containing at least eight different samples and always an equal amount of both RNA and RPF libraries. Sample pools were sequenced on the HiSeq 2500 platform using 50-bp sequencing chemistry.

The Ribo-Taq mouse model and ribosome-protected fragments immunoprecipitation were described previously (Doroudgar et al, 2019). To identify mTOR-dependent translatome in vitro polysome fractionations from NRCMs were isolated in a sucrose gradient according to 440 standard procedure (Kmietczyk et al, 2019). The absorbance was monitored at $254 \mathrm{~nm}$ to 
bioRxiv preprint doi: https://doi.org/10.1101/2020.11.29.403196; this version posted December 1, 2020. The copyright holder for this preprint (which was not certified by peer review) is the author/funder. All rights reserved. No reuse allowed without permission.

record the polysome profile. The collected fractions were treated with RNasel and mixed with Qiazol to recover ribosomal footprints. Generation of Ribo- and RNA-seq libraries and deepsequencing data processing were described in detail(Doroudgar et al, 2019). Briefly, 15 Million NRCMs were lysed in $500 \mu l$ polysome buffer $(20 \mathrm{mM}$ Tris pH 7.4, $10 \mathrm{mM} \mathrm{MgCl}, 200 \mathrm{mM} \mathrm{KCl}$,

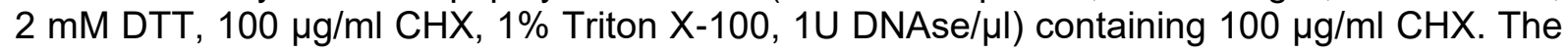
lysate was used for RPF generation using polysome profiles after RNAse1 digestion. For complete lysis, the samples were kept on ice for $10 \mathrm{~min}$ and subsequently centrifuged at $20,000 \times g$ to precipitate cell debris and the supernatant was immediately used in the further steps. Sucrose solutions were prepared in polysome gradient buffer and $20 \mathrm{U} / \mathrm{mL}$ SUPERaseIn (Ambion). Sucrose density gradients (10-50\% wt/vol) were freshly made in SW40 ultracentrifuge tube using a BioComp Gradient Master (BioComp). Ribosome footprints were generated after treating the lysate with RNAse I (Ambion). Cell lysates were loaded onto sucrose gradients, followed by centrifugation for $250 \mathrm{~min}$ at $220,000 \times \mathrm{g}, 4^{\circ} \mathrm{C}$, in an SW40 rotor. Separated samples were fractionated at $0.375 \mathrm{ml} / \mathrm{min}$ by using a fractionation system BioComp Gradient Station (BioComp) that continually monitors OD254 values. Monosomal fractions were collected into tubes at $0.3 \mathrm{~mm}$ intervals. Libraries were generated according to the mammalian Ribo-seq kit (Illumina). Sample pools were sequenced on the HiSeq 2000 platform using 50-bp sequencing chemistry.

\section{Data analysis and visualization.}

For RNA-Seq R and Bioconductor was used with the NGS analysis package systempipeR (Backman \& Girke, 2016). Quality control of raw sequencing reads was performed using FastQC. Low-quality reads were removed using trim_galore (version 0.6.4). The resulting reads were aligned to mouse genome version mm10 from UCSC using tophat2 (Kim et al, 2013). EdgeR (version 3.26.8) was used to perform a differential expression analysis (Robinson et al, 2010). A false-positive rate of $\alpha=0.05$ with FDR correction was taken as the level of significance. Volcano plots were created using $R$ (version 3.4.0) using the ggplot2 package (version 2.2.1).

For Ribo-seq data, only periodic fragment lengths were kept that showed a distinctive triplet periodicity. We used the automatic Bayesian selection of read lengths and ribosome P-site offsets (BPPS) method (Malone et al, 2016) to select and shift aligned reads to properly account for the P-site of the ribosome. We only consider data points with read count observations across all replicates. We used log2Fc $>1.5$ as a cutoff. For the myocytes data we do not estimate significance but provide only fold change values due to the absence of biological replicates.

GO-term enrichment analysis was performed using DAVID (Database for Annotation, Visualization and Integrated Discovery) with the rat genome as background. Only enriched GO terms with at least three significantly changed genes were kept for further analysis and ranked by Fisher Exact. Top enriched terms were retained and visualized with a custom plotting routine showing enrichment $p$-value.

\section{Mice, Surgery, and cardiac function}

To study Cand2 in vivo, a novel Cand2 knock-out mouse line was created. An embryonic stem cell clone (EPD0169_1_A01-European Conditional Mouse Mutagenesis Program) was used to generate Cand2 knock-out mice. At 8 weeks of age, male Cand2 ${ }^{-/}$mice underwent transverse aortic constriction (TAC; 27 gauge needle) or sham operation, as previously described (Kmietczyk et al, 2019;). Institutional Animal Care and Use Committee approval was obtained for all animal studies.

\section{Isolation and primary culture of neonatal and adult ventricular cardiomyocytes and neonatal mouse cardiomyocytes.}

Ventricular cardiomyocytes from 1 to 2 day old Wistar rats neonatal hearts (NRCMs) were prepared by trypsin digestion and percoll gradient separation according to standard procedures (Sanlialp et al, 2020). For analysis of hypertrophy, cells were treated with $50 \mathrm{uM}$ $\mathrm{PE}$ for indicated time points. mTORC1 was pharmacologically inhibited with $250 \mathrm{nM}$ Torin1 for 
bioRxiv preprint doi: https://doi.org/10.1101/2020.11.29.403196; this version posted December 1, 2020. The copyright holder for this preprint (which was not certified by peer review) is the author/funder. All rights reserved. No reuse allowed without permission.

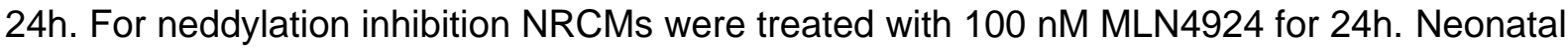
mouse heart myocytes were isolated from 1-day old wild-type and Cand2 knock-out mice by DNasel/collagenase type III digestion according to the published protocol (Ehler et al, 2013). Adult ventricular cardiomyocytes were isolated using standard procedures as previously described (Kmietczyk et al, 2019).

\section{Plasmids and overexpressions.}

5'TOP-reporter constructs were obtained by cloning of Cand2, eEF2, and -Actin 5'UTRs into piCheck2 plasmid upstream of Renilla luciferase. HEK cells were transfected with 5'UTRreporters for $24 \mathrm{~h}$ using ViaFect reagent according to the manufacturer's protocol (Promega). Next, HEK cells were treated with $250 \mathrm{nM}$ Torin1 for $6 \mathrm{~h}$. Cand2 overexpression in vitro was obtained by NRCMs transduction with AAV6 vector. Mouse Cand2 (NM 025958.2) containing Myc-tag inserted after START codon was chemically synthesized (BioCat) and cloned into recipient vector pSSV9. To determine the optimal MOI, a preliminary dose-escalation experiment was conducted with different doses of Cand2 AAV6. For MEF2 and Grk5 studies, NRCMs were infected with adenoviruses harboring 3xMEF-luc (Firefly luciferase; a gift from Prof. Johannes Backs) and bovine Grk5 (gift from Prof. Philip Raake) for 24h.

\section{RNA interference.}

Pre-designed synthetic anti-rat Cand2 small interfering RNA (siRNA ID s140407), anti-Cul1 siRNA (s168977), anti-Rptor siRNA (s143005) and scrambled siRNA (Silencer ${ }^{\mathrm{TM}}$ Select Negative Control No. 1 siRNA) as negative control were purchased in Thermo Fisher Scientific. NRCMs were transfected with $25 \mathrm{nM}$ final concentration of siRNAs by using HiPerfect transfection reagent according to the manufacturer's instructions (QIAGEN).

\section{RNA isolation and RT-qPCR.}

Total RNA from NRCMs was isolated with Quick-RNA ${ }^{\text {TM }}$ MiniPrep (Zymo Research) according to the manufacturer's protocol. Liquid nitrogen snap-frozen tissues were homogenized in Precellys 24 homogenizer (Bertin Instruments) in 500 ul of lysis buffer containing $20 \mathrm{mM}$ Hepes $\mathrm{pH}$ 7.4-7.5, $10 \mathrm{mM} \mathrm{MgCl}$, $200 \mathrm{mM} \mathrm{KCl}, 1 \%$ Triton, 1x protease inhibitor, 1x phosphatase inhibitor, $25 \mathrm{U} / \mu \mathrm{L}$ DNase I and $40 \mathrm{U}$ of RNasin. A $100 \mathrm{ul}$ aliquot was mixed with $1 \mathrm{ml}$ of Qiazol to isolate total RNA according to the standard protocols with chloroform and isopropanol precipitation. The quality of total RNA was checked on a NanoChip 2100 Bioanalyzer (Agilent). 100-500 ng of total RNA was reverse-transcribed into complementary DNA (cDNA) by using iScript $^{\mathrm{TM}}$ Reverse Transcription Supermix (Biorad). Quantitative real-time PCR was performed using iTAQ $^{\text {TM }}$ SYBR Green PCR Kit (Biorad) according to the manufacturer's instructions. Primers used in the study are shown in supplementary Table 1. Analysis of the specificity of the amplification product was performed by melting curve analysis. We calculated quantitative differences using the $\Delta \Delta C(T)$ method.

\section{Immunoblots}

Samples were combined with the appropriately concentrated form of Laemmli sample buffer and then boiled before SDS-PAGE followed by transfer to PVDF membranes.

A list of all used antibodies is provided in Table 2.

\section{Histology, immunohistochemistry, and PLA.}

Sections were cut and deparaffinized using standard procedures. In brief, hearts were excised and embedded in formalin for 24 hours at room temperature. After cutting, sections were deparaffinized, rehydrated and antigens were retrieved.

NRCMs and ARCMs were plated on permanox or glass chamber slides (gelatin coated) and fixed by paraformaldehyde, permeabilized and blocked as described (Völkers et al, 2013). Cand2 primary antibody (Bethyl Laboratories) diluted 1:100 vol/vol in the respective blocking solution was applied to both types of slides overnight at $4^{\circ} \mathrm{C}$. Cand2 was detected by FITC or Cy3-conjugated secondary antibody (Jackson Laboratories). Alexa Fluor ${ }^{\mathrm{TM}} 555$ Phalloidin (Thermo Fisher Scientific) or conjugated phalloidin 633 (Jackson Laboratories) was used to 
detect F-actin. DAPI (Life Technologies) was diluted in Vectashield (Vectra Labs) mounting media and used as nuclear staining.

Proximity ligation assay was performed according to the manufacturer's instructions (DuoLink in situ red kit Mouse/Rabbit) in rat and mouse neonatal cardiomyocytes. Primary antibodies were diluted 1:20 in DuoLink antibody diluent and incubated with cells at $4^{\circ} \mathrm{C}$ overnight. For Cand2-Grk5 detection anti-rabbit TIP120B and anti-mouse Grk5 were used. For Cand2-Cul1 anti-mouse TIP120B and anti-rabbit Cul1 antibodies were applied. The connective tissue was visualized with WGA Alexa Fluor ${ }^{\mathrm{TM}} 488$ conjugate (Thermo Fisher Scientific). Images were obtained with $20 x$ and $60 x$ objectives.

\section{Immunoprecipitation.}

NRCMs were treated with 1 uM MLN 4924 for $6 \mathrm{~h}$ and lysed with ice-cold IP lysis buffer containing $40 \mathrm{mM}$ Hepes $\mathrm{pH} 7.4,2 \mathrm{mM}$ EDTA, $10 \mathrm{mM}$ sodium pyrophosphate, $10 \mathrm{mM}$ glycerophosphate, and $0.3 \%$ CHAPS. M280 sheep anti-rabbit Dynabeads were coated with anti-rabbit TIP120B antibody in IP lysis buffer for $1 \mathrm{~h}$ at room temperature. $500 \mathrm{ug}$ of protein lysate was combined with antibody-coated beads and rotated at $4{ }^{\circ} \mathrm{C}$ overnight. Beads were washed three times with IP lysis buffer supplemented with $150 \mathrm{mM} \mathrm{NaCl}$. Immunocomplexes were eluted with SDS sample buffer by boiling for $5 \mathrm{~min}$, and proteins were detected by immunoblotting with a corresponding antibody.

564

565

566

567

568

569

570

571

572

573

574

575

576

577

578

579

580

\section{Subcellular fractionation.}

Subcellular fractionation of $\mathrm{C} 2 \mathrm{C} 12$ cells and left ventricles was performed as previously described with small modifications (Wysocka et al, 2001). Briefly, cells and tissues were washed with ice-cold PBS and homogenized in buffer A $(10 \mathrm{mM}$ Hepes $\mathrm{pH} 7.9,10 \mathrm{mM} \mathrm{KCl}$, $1.5 \mathrm{mM} \mathrm{MgCl}_{2}, 340 \mathrm{mM}$ sucrose, $10 \%$ glycerol, $1 \mathrm{mM} \mathrm{DTT}, 1 \mathrm{x}$ proteases inhibitor cocktails, $1 \mathrm{X}$ Triton) with tight-fitting disposable tissue grinder pestle and needled with 20 and 22 gauge needles. Nuclear pellets and cytoplasmic supernatants were separated by low-speed centrifugation at $1300 \times \mathrm{g}, 4^{\circ} \mathrm{C}$ for $5 \mathrm{~min}$. The final cytoplasmic fraction was pre-cleared by high-speed centrifugation at $20000 \times \mathrm{g}$ for $5 \mathrm{~min}$ at $4^{\circ} \mathrm{C}$. The nuclear pellet from low-speed centrifugation was dissolved in the hypotonic buffer $B$ containing 3 mM EDTA, 0.2 mM EGTA, $1 \mathrm{mM}$ DTT, $1 \mathrm{x}$ proteases inhibitor cocktails.

\section{Luciferase Assays.}

Cells were harvested 24-48 h after transfection/infection in passive lysis buffer (Promega). The samples were prepared according to the manufacturer's protocol (Promega) and measured by using a LUMIstar OPTIMA plate reader. Luciferase's signals were normalized to total protein amount determined with $R C-D C$ kit (BioRad).

Table 1. List of primers

\begin{tabular}{|c|c|c|}
\hline Gene & Forward (5'-3') & Reverse (5'-3') \\
\hline Rat Nppa & TACAGTGCGGTGTCCAACACAGAT & TGGGCTCAATCCTGTCAATCCTA \\
\hline Rat Nppb & GAACAATCCATGATGCAGAAGC & GCTGTCTCTGAGCCATTTCCT \\
\hline Rat Grk5 & CTCTTCAGGCGTCAGCATCA & AGGCCAGAGCTGAAACTAGC \\
\hline Rat Cand2 & CAAAGACTTCAGGTTCATGGCTAC & GTTCTGCACCTCACCACTCC \\
\hline Rat Nr4a1 & CTTCAAAACCCAAGCAGCCC & CTTCAAAACCCAAGCAGCCC \\
\hline Rat Xirp2 & TCGCCTACAAACCTCAGACG & $\begin{array}{l}\text { ACAGTTTCACTCAAAAAGGCCA } \\
\text { A }\end{array}$ \\
\hline Rat Raptor & AGAGCTGGAGAATGAAGGATCG & AGGACCCATAGACAGAGGATCAAT \\
\hline $\begin{array}{l}\text { Rat, mouse } \\
\text { HPRT }\end{array}$ & GGGGCTGTACTGCTTAACCAG & TCAGTCAACGGGGGACATAAA \\
\hline Mouse Cand2 & GGGCAAGGTGAAGGAGTACC & TGGTGAGTTGGCCTGTGATC \\
\hline Rat, mouse 18S & CGAGCCGCCTGGATACC & CATGGCCTCAGTTCCGAAAA \\
\hline Mouse Grk5 & CTCCGAAGGACCATAGACAGAG & CGCCCCAAGGTTTTCATCTG \\
\hline Mouse Xirp2 & GCAGCTTCTCGGCTAATGTCA & CTCAGAAAAGGCGTTGCAGG \\
\hline
\end{tabular}




\begin{tabular}{|l|l|l|}
\hline Mouse Nppa & TTGTGGTGTGTCACGCAGCT & TGTTCACCACGCCACAGTG \\
\hline Mouse Nppb & TTTGGGCTGTAACGCACTG & CACTTCAAAGGTGGTCCCAGA \\
\hline Mouse Cand1 & GACGACGATGACCAAGGGAG & TAACTACAGCGTCCAGGCAC \\
\hline Mouse Desmin & AGTGCATGAAGAGGAGATCCG & GTTCTTAGCCGCGATGGTCT \\
\hline Mouse 4EBP1 & ACTCACCTGTGGCCAAAACA & TTGTGACTCTTCACCGCCTG \\
\hline Bovine Grk5 & CTGTTAGACGATTACGGCCACA & GGAGCCATGTAGCCAACGG \\
\hline
\end{tabular}

581 Table 2. List of primary antibodies

\begin{tabular}{|l|l|l|l|l|l|}
\hline Antigen & Primary antibody & Origin & Company & Cat.\# & Application \\
\hline Cand2 & TIP120B & Rabbit & $\begin{array}{l}\text { Bethyl } \\
\text { Laboratories }\end{array}$ & $\begin{array}{l}\text { A304-046A- } \\
\text { M }\end{array}$ & $\begin{array}{l}\text { WB, IP, IF, } \\
\text { PLA }\end{array}$ \\
\hline Cand2 & TIP120B (H7) & Mouse & Santa Cruz & sc-515406 & WB, PLA \\
\hline Grk5 & Grk5 (D9) & Mouse & Santa Cruz & sc-518005 & WB, PLA \\
\hline Cul1 & Cul1 (D5) & Mouse & Santa Cruz & sc-17775 & WB, PLA \\
\hline Cul1 & Cul1 & Rabbit & $\begin{array}{l}\text { Bethyl } \\
\text { Laboratories }\end{array}$ & $\begin{array}{l}\text { A303-373A- } \\
\text { M }\end{array}$ & WB, PLA \\
\hline GAPDH & GAPDH (G9) & Mouse & Santa Cruz & sc-365062 & WB \\
\hline$\beta-$ Actin & Beta-Actin (C4) & Mouse & Santa Cruz & sc-47778 & WB \\
\hline pRib6S & pS6RP (Ser235/236) & Rabbit & Cell Signaling & $4858 S$ & WB \\
\hline p4E-BP1 & p4E-BP1 (Ser65) & Rabbit & Cell Signaling & $9451 S$ & WB \\
\hline 4E-BP & 4E-BP & Rabbit & Cell Signaling & 9452 & WB \\
\hline pERK 1/2 & Thr218/Tyr220 & Rabbit & Cell Signaling & 3371 & WB \\
\hline Myc-Tag & (71D10) & Rabbit & Cell Signaling & $2278 S$ & WB \\
\hline pAkt1 & Ser473 & Rabbit & Cell Signaling & 9271 & WB \\
\hline Csq & calsequestrin & Rabbit & abcam & Ab3516 & WB \\
\hline rpS5 & rpS5 & Rabbit & abcam & Ab210745 & WB \\
\hline Lamin B1 & Lamin B1 (B10) & Mouse & Santa Cruz & sc-374015 & WB \\
\hline $\begin{array}{l}\text { Histone1 } \\
\text { H3D }\end{array}$ & Histone1 H3D & Mouse & Santa Cruz & sc-134355 & WB \\
\hline Cul4 & Cul4 (H11) & Mouse & Santa Cruz & sc-377188 & WB \\
\hline Des & Desmin & Rabbit & Abcam & ab15200 & WB \\
\hline eEF2 & eEF2 & Rabbit & Cell Signaling & $2332 s$ & WB \\
\hline rpS20 & rpS20 & Rabbit & Abcam & ab133776 & WB \\
\hline
\end{tabular}

582

583

584

585

586

587

588

589

590

591

592

593

594

595

596

\section{Statistics}

In vivo experiments were performed on 3-20 biological replicates (mice) for each treatment. Throughout the studies, the investigators were blinded to the sample group allocation during the experiment and analysis of the experimental outcome. Statistical analysis was performed using GraphPad Prism 7.0 (Graphpad Software Inc; www.graphpad.com) or R. All the data sets were tested for normality of distribution using the Shapiro-Wilks test (threshold $P<0.05)$. For normally distributed data, values shown are mean \pm SEM. Statistical analysis of data involving two groups was performed using unpaired two-tailed t-test, for more than two groups 1-way ANOVA with the Bonferroni test applied to correct for multiple comparisons.Sequencing count data were modeled using negative-binomial distribution. For not normally distributed data a nonparametric test was used to test for significance between different groups. A Mann-Whitney test was performed when comparing two groups. A Kruskal-Wallis test was used when comparing multiple groups (more than two) followed by a Dunn's multiple test comparison. 
Data Availability Section

Raw sequencing data have been made publicly available and can be accessed.

RNA-Seq: Raw data have been uploaded to GEO (accession ID: GSE153364)

Ribo-Seq: Raw data have been uploaded to SRA (accession ID:SRP156230)

\section{Acknowledgements}

A.A.G., H.A.K., M.V., S.D., and C.D. acknowledge the DZHK (German Center for Cardiovascular Research) Partner Site Heidelberg/Mannheim. C.D. acknowledges funding from the Klaus-Tschira Stiftung GmbH. S.D. acknowledges the European Society of Cardiology Basic Research Fellowship and the DZHK Excellence Programme. M.V. acknowledges the DFG (German Research Foundation, DFG VO 1659 2/1, DFG VO 1659 2/2, DFG VO 1659 4/1, DFG VO 1659 6/1), the Boehringer Ingelheim Foundation (Plus 3 Programme).

\section{Disclosures}

All authors declare no competing financial interests.

\section{References:}

Aoki T, Okada N, Ishida M, Yogosawa S, Makino Y \& Tamura TA (1999) TIP120B: A novel TIP120-family protein that is expressed specifically in muscle tissues. Biochem Biophys Res Commun

Avni D, Biberman Y \& Meyuhas O (1996) The 5' Terminal Oligopyrimidine Tract Confers Translational Control on Top Mrnas in a Cell Type-and Sequence Context-Dependent Manner. Nucleic Acids Res 25: 995-1001

Backman TWH \& Girke T (2016) systemPipeR: NGS workflow and report generation environment. BMC Bioinformatics 17: 388

Buss SJ, Muenz S, Riffel JH, Malekar P, Hagenmueller M, Weiss CS, Bea F, Bekeredjian R, Schinke-Braun M, Izumo S, et al (2009) Beneficial Effects of Mammalian Target of Rapamycin Inhibition on Left Ventricular Remodeling After Myocardial Infarction. J Am Coll Cardiol 54: 2435-2446

Cannavo A, Liccardo D, Eguchi A, Elliott KJ, Traynham CJ, Ibetti J, Eguchi S, Leosco D, Ferrara N, Rengo G, et al (2016) Myocardial pathology induced by aldosterone is dependent on non-canonical activities of $\mathrm{G}$ protein-coupled receptor kinases. Nat Commun 7

Doroudgar S, Hofmann C, Boileau E, Malone B, Riechert E, Gorska AA, Jakobi T, Sandmann C, Jürgensen L, Kmietczyk V, et al (2019) Monitoring cell-Type-specific gene expression using ribosome profiling in vivo during cardiac hemodynamic stress. Circ Res 125: 431-448

Drews O \& Taegtmeyer $\mathrm{H}$ (2014) Targeting the ubiquitin-proteasome system in heart disease: The basis for new therapeutic strategies. Antioxidants Redox Signal 21: 23222343 doi:10.1089/ars.2013.5823 [PREPRINT]

Ehler E, Moore-Morris T \& Lange S (2013) Isolation and culture of neonatal mouse cardiomyocytes. J Vis Exp

Furukawa M, Zhang Y, McCarville J, Ohta T \& Xiong Y (2000) The CUL1 C-Terminal Sequence and ROC1 Are Required for Efficient Nuclear Accumulation, NEDD8 Modification, and Ubiquitin Ligase Activity of CUL1. Mol Cell Biol 20: 8185-8197 
Gold JI, Martini JS, Hullmann J, Gao E, Chuprun JK, Lee L, Tilley DG, Rabinowitz JE, Bossuyt J, Bers DM, et al (2013) Nuclear Translocation of Cardiac G Protein-Coupled Receptor Kinase 5 Downstream of Select Gq-Activating Hypertrophic Ligands Is a Calmodulin-Dependent Process. PLoS One 8

Goldenberg SJ, Cascio TC, Shumway SD, Garbutt KC, Liu J, Xiong Y \& Zheng N (2004) Structure of the Cand1-Cul1-Roc1 complex reveals regulatory mechanisms for the assembly of the multisubunit cullin-dependent ubiquitin ligases. Cell 119: 517-528

Gregers E, Ahlberg G, Christensen T, Jabbari J, Larsen KO, Herfelt CB, Henningsen KM, Andreasen L, Thiis JJ, Lund J, et al (2017) Deep sequencing of atrial fibrillation patients with mitral valve regurgitation shows no evidence of mosaicism but reveals novel rare germline variants. Hear Rhythm 14: 1531-1538

van Heesch S, Witte F, Schneider-Lunitz V, Schulz JF, Adami E, Faber AB, Kirchner M, Maatz H, Blachut S, Sandmann CL, et al (2019) The Translational Landscape of the Human Heart. Cell 178: 242-260.e29

Hsieh AC, Liu Y, Edlind MP, Ingolia NT, Janes MR, Sher A, Shi EY, Stumpf CR, Christensen C, Bonham MJ, et al (2012) The translational landscape of mTOR signalling steers cancer initiation and metastasis. Nature 485: 55-61

Huang HT, Brand OM, Mathew M, Ignatiou C, Ewen EP, McCalmon SA \& Naya FJ (2006) Myomaxin Is a novel transcriptional target of MEF2A that encodes a Xin-related $\alpha-$ actinin-interacting protein. J Biol Chem 281: 39370-39379

ladevaia V, Zhang Z, Jan E \& Proud CG (2012) MTOR signaling regulates the processing of pre-rRNA in human cells. Nucleic Acids Res 40: 2527-2539

Ingolia NT, Ghaemmaghami S, Newman JRS \& Weissman JS (2009) Genome-wide analysis in vivo of translation with nucleotide resolution using ribosome profiling. Science (80-) 324: 218-223

Jefferies HBJ, Reinhard C, Kozma SC \& Thomas G (1994) Rapamycin selectively represses translation of the 'polypyrimidine tract' mRNA family. Proc Natl Acad Sci U S A 91: 4441-4445

Johnson LR, Scott MGH \& Pitcher JA (2004) G Protein-Coupled Receptor Kinase 5 Contains a DNA-Binding Nuclear Localization Sequence. Mol Cell Biol 24: 10169-10179

Kim D, Pertea G, Trapnell C, Pimentel H, Kelley R \& Salzberg SL (2013) TopHat2: Accurate alignment of transcriptomes in the presence of insertions, deletions and gene fusions. Genome Biol 14: R36

Kmietczyk V, Riechert E, Kalinski L, Boileau E, Malovrh E, Malone B, Gorska A, Hofmann C, Varma E, Jürgensen L, et al (2019) M 6 A-mRNA methylation regulates cardiac gene expression and cellular growth. Life Sci Alliance 2: e201800233

Lehmann LH, Jebessa ZH, Kreusser MM, Horsch A, He T, Kronlage M, Dewenter M, Sramek $\mathrm{V}$, Oehl U, Krebs-Haupenthal J, et al (2018) A proteolytic fragment of histone deacetylase 4 protects the heart from failure by regulating the hexosamine biosynthetic pathway. Nat Med 24: 62-72

Liu J, Furukawa M, Matsumoto T \& Xiong Y (2002) NEDD8 modification of CUL1 dissociates p120CAND1, an inhibitor of CUL1-SKP1 binding and SCF ligases. Mol Cell 10: 15111518 
Liu X, Reitsma JM, Mamrosh JL, Zhang Y, Straube R \& Deshaies RJ (2018) Cand1Mediated Adaptive Exchange Mechanism Enables Variation in F-Box Protein Expression. Mol Cell 69: 773-786.e6

Ma XM \& Blenis J (2009) Molecular mechanisms of mTOR-mediated translational control. Nat Rev Mol Cell Biol 10: 307-318

Malone B, Atanassov I, Aeschimann F, Li X, Großhans H \& Dieterich C (2016) Bayesian prediction of RNA translation from ribosome profiling. Nucleic Acids Res 45: 2960-2972

Martini JS, Raake P, Vinge LE, DeGeorge BR, Chuprun JK, Harris DM, Gao E, Eckhart AD, Pitcher JA \& Koch WJ (2008) Uncovering G protein-coupled receptor kinase-5 as a histone deacetylase kinase in the nucleus of cardiomyocytes (Proceedings of the National Academy of Sciences of the United States of America (2008) 105, (1245712462) DOI: 10.1073/pnas.0803153105). Proc Natl Acad Sci U S A 105: 17206

Min KW, Kwon MJ, Park HS, Park Y, Sungjoo KY \& Yoon JB (2005) CAND1 enhances deneddylation of CUL1 by COP9 signalosome. Biochem Biophys Res Commun 334: 867-874

Morihara H, Yamamoto T, Oiwa H, Tonegawa K, Tsuchiyama D, Kawakatsu I, Obana M, Maeda M, Mohri T, Obika S, et al (2017) Phospholamban Inhibition by a Single Dose of Locked Nucleic Acid Antisense Oligonucleotide Improves Cardiac Contractility in Pressure Overload-Induced Systolic Dysfunction in Mice. J Cardiovasc Pharmacol Ther 22: 273-282

Poulin F, Gingras AC, Olsen H, Chevalier S \& Sonenberg N (1998) 4E-BP3, a new member of the eukaryotic initiation factor 4E-binding protein family. J Biol Chem 273: 1400214007

Robinson MD, Mccarthy DJ \& Smyth GK (2010) edgeR: a Bioconductor package for differential expression analysis of digital gene expression data

Sanlialp A, Schumacher D, Kiper L, Varma E, Riechert E, Ho TC, Hofmann C, Kmietczyk V, Zimmermann F, Dlugosz S, et al (2020) Saraf-dependent activation of mTORC1 regulates cardiac growth. $J$ Mol Cell Cardiol 141: 30-42

Sciarretta S, Forte M, Frati G \& Sadoshima J (2018) New insights into the role of mtor signaling in the cardiovascular system. Circ Res 122: 489-505

Shioi T, McMullen JR, Tarnavski O, Converso K, Sherwood MC, Manning WJ \& Izumo S (2003) Rapamycin attenuates load-induced cardiac hypertrophy in mice. Circulation 107: $1664-1670$

Shiraishi S, Zhou C, Aoki T, Sato N, Chiba T, Tanaka K, Yoshida S, Nabeshima Y, Nabeshima Y \& Tamura T (2007) TBP-interacting Protein 120B (TIP120B)/Cullinassociated and Neddylation-dissociated 2 (CAND2) Inhibits SCF-dependent Ubiquitination of Myogenin and Accelerates Myogenic Differentiation. J Biol Chem 282: 9017-9028

Sinner MF, Tucker NR, Lunetta KL, Ozaki K, Smith JG, Trompet S, Bis JC, Lin H, Chung MK, Nielsen JB, et al (2014) Integrating genetic, transcriptional, and functional analyses to identify 5 novel genes for atrial fibrillation. Circulation 130: 1225-1235

Su H, Li J, Menon S, Liu J, Kumarapeli AR, Wei N \& Wang X (2011) Perturbation of cullin deneddylation via conditional Csn8 ablation impairs the ubiquitin-proteasome system 
and causes cardiomyocyte necrosis and dilated cardiomyopathy in mice. Circ Res 108: 40-50

Su H, Li J, Osinska H, Li F, Robbins J, Liu J, Wei N \& Wang X (2013) The COP9 Signalosome Is Required for Autophagy, Proteasome-Mediated Proteolysis, and Cardiomyocyte Survival in Adult Mice. Circ Hear Fail 6: 1049-1057

Thoreen CC, Chantranupong L, Keys HR, Wang T, Gray NS \& Sabatini DM (2012) A unifying model for mTORC1-mediated regulation of mRNA translation. Nature 485: 109-113

Traynham CJ, Hullmann J \& Koch WJ (2016) 'Canonical and non-canonical actions of GRK5 in the heart'. J Mol Cell Cardiol 92: 196-202 doi:10.1016/j.yjmcc.2016.01.027 [PREPRINT]

Uprety B, Kaja A \& Bhaumik SR (2018) TOR Facilitates the Targeting of the 19S Proteasome Subcomplex To Enhance Transcription Complex Assembly at the Promoters of the Ribosomal Protein Genes. Mol Cell Biol 38: 1-17

Völkers M, Doroudgar S, Nguyen N, Konstandin MHH, Quijada P, Din S, Ornelas L, Thuerauf DJJ, Gude N, Friedrich K, et al (2014) PRAS40 prevents development of diabetic cardiomyopathy and improves hepatic insulin sensitivity in obesity. EMBO Mol Med 6: 57-65

Wysocka J, Reilly PT \& Herr W (2001) Loss of HCF-1-Chromatin Association Precedes Temperature-Induced Growth Arrest of tsBN67 Cells. Mol Cell Biol 21: 3820-3829

Zhang D, Contu R, Latronico MVG, Zhang JL, Rizzi R, Catalucci D, Miyamoto S, Huang K, Ceci M, Gu Y, et al (2010) MTORC1 regulates cardiac function and myocyte survival through 4E-BP1 inhibition in mice. J Clin Invest 120: 2805-2816

Zhang Y, Matkovich SJ, Duan X, Gold JI, Koch WJ \& Dorn GW (2011) Nuclear effects of Gprotein receptor kinase 5 on histone deacetylase 5-regulated gene transcription in heart failure. Circ Hear Fail 4: 659-668

Zheng N, Schulman BA, Song L, Miller JJ, Jeffrey PD, Wang P, Chu C, Koepp DM, Elledge SJ, Paganok M, et al (2002) Structure of the Cul1-Rbx1-Skp1-F box Skp2 SCF ubiquitin ligase complex. Nature 416: 703-709

Zou J, Ma W, Li J, Littlejohn R, Zhou H, Kim I man, Fulton DJR, Chen W, Weintraub NL, Zhou J, et al (2018) Neddylation mediates ventricular chamber maturation through repression of Hippo signaling. Proc Natl Acad Sci U S A 115: E4101-E4110

Zou J, Ma W, Littlejohn R, Li J, Stansfield BK, Kim IM, Liu J, Zhou J, Weintraub NL \& Su H (2019) Transient inhibition of neddylation at neonatal stage evokes reversible cardiomyopathy and predisposes the heart to isoproterenol-induced heart failure. $\mathrm{Am} \mathrm{J}$ Physiol - Hear Circ Physiol 316: H1406-H1416 
bioRxiv preprint doi: https://doi.org/10.1101/2020.11.29.403196; this version posted December 1, 2020. The copyright holder for this preprint (which was not certified by peer review) is the author/funder. All rights reserved. No reuse allowed without permission.

771

772 
Figures legends.

\section{Figure 1. Identification of the mTORC1-dependent cardiac translatome}

(A) Experimental design of translational profiling of mTOR-dependent mRNAs in vitro in NRCMs. Polysomal fractions were isolated in a sucrose gradient and ribosomal footprints were recovered by ribonuclease digestion. NRCMs were treated with vehicle or Torin1 to block mTOR pathway. (B) Scatter plot of ribosome occupancy in Ribo-seq of cultured cardiac myocytes in response to PE and Torin 1 treatment (blue dots - mRNAs downregulated after Torin 1, red dots - upregulated mRNA. (C) Percentage of TOP or TOP-like containing mRNAs in total and Torin1-sensitive mRNA pools. (D) Transcriptional (RNA-seq) vs. Translational (Ribo-seq) control of mTOR-regulated mRNAs. (E) Network of enriched GO categories (biological process) among mTOR-sensitive genes into several functional groups represented by circles of different colors. Each node represents one enriched GO category with the size of the node is proportional to the number of transcripts. Edges indicate similarity (between the two connected GO categories). (F) Cumulative fraction of mRNAs relative to their fold change of Ribo-seq and (G) RNA-seq (TAC vs. sham) between all transcripts mTOR-dependent and independent transcripts two days after TAC surgery. (H) Immunoblot and (I) RT-qPCR representing levels of proteins and corresponding mRNAs with defined 5'TOP motifs 3 hours and 2 days after TAC.

Figure 2. Cand2 is translationally upregulated during cardiac pathological stress.

(A) Venn diagram showing a group of transcripts translationally upregulated in vivo 2 days after TAC and regulated by mTORC1 in vitro in NRCMs treated with Torin1. Table with top the most translationally regulated mTOR-dependent transcripts in vitro after Torin1 treatment of NRCMs and in vivo after TAC (B) Scatter plot of Ribo-seq vs. RNA-seq upon NRCMs treatment of Torin1 shows Cand2 among translationally downregulated mRNAs. (C) Protein expression of Cand2 in NRCMs treated with PE for 3 and $24 \mathrm{~h}$, and $24 \mathrm{~h}$ of Torin 1 treatment. mTORC1 induction was monitored by downstream targets, p4EBP1 and pS6K proteins. rpS5 - example of mTOR-dependent mRNA used as a positive control. (D) Cand2 mRNA levels in NRCMs treated with PE and Torin1 measured by RT-qPCR. (E) Scatter plot of Ribo-seq vs. RNA-seq 2 days after TAC showing an increase of Cand2 translation (F) Ribo-seq and (G) RNA-seq expression data of Cand2 in sham and TAC operated animals (CPM - count per million). (H) Immunoblots of Cand2 expression in left ventricles 2 days postTAC and after mice injection with Torin1. rpS5 used as a positive control of mTOR-dependent mRNA. (I) Cand2 mRNA 2 days after TAC measured by RT-qPCR. (J) Schematic representation of reporter consisting of human Cand2 5'UTR and downstream Renilla luciferase coding sequence. Potential TOP-like motif is highlighted in a grey box. Grey arrows indicate transcription start sites in the adult heart according to Database of Transcriptional Start Sites (Release 10.1). Cand2 5'UTR effect on translation measured by luciferase activity in normal conditions and after mTORC1 block with Torin1. eEF2 - positive control of reporter with defined 5'TOP motif and non-TOP 5'UTR of $\beta$-Actin used as a negative control. Total protein content in lysates used for luciferase assay.

\section{Figure 3. Cand2 is a muscle-specific protein}

(A) Representative immunoblot of Cand2 protein levels in mouse organs. (B) Cand2 expression in mouse organs measured by RT-qPCR. (C) Immunofluorescent staining of Cand2 (red), actin (green) and nuclei (DAPI, blue) in paraffin sections from left ventricles of WT and Cand2 KO mice. Scale bar $20 \mu \mathrm{m}$ (D) Immunofluorescence of adult rat cardiomyocytes of Cand2 (red), sarcomeric actin (green), and nuclei (blue). Scale bar $50 \mu \mathrm{m}$. (E) Immunoblot of subcellular fractionation of left ventricle (LV). Gapdh and Histone 3D (H3D) used as cytoplasmic and nuclear markers, respectively. WCL - whole cell lysate, cyt - cytoplasmic fraction, nuc - nuclear fraction. 
bioRxiv preprint doi: https://doi.org/10.1101/2020.11.29.403196; this version posted December 1, 2020. The copyright holder for this preprint (which was not certified by peer review) is the author/funder. All rights reserved. No reuse allowed without permission.

\section{Figure 4. Cand2 is required for cardiac hypertrophy induction.}

(A) Representative immunoblot of Cand2 KD in NRCMs with Cand2 specific siRNA (siScr control scrambled siRNA). (B) Cand2 OE in NRCMs (Cand2 AAV6; Ctr AAV - control AAV6 vector) analyzed by Western blot. $\beta$-Actin - housekeeping protein. (C). Cell surface area measurement of NRCM after Cand2 KD and 24h PE stimulation (D). RT-qPCR analysis of Nppa transcript level after Cand2 KD. (E) NRCMs size measurement after Cand2 OE and 24h PE stimulation. (F) Nppa mRNA levels in vitro after Cand2 OE and PE treatment measured by RT-qPCR. (G) Ejection fraction 2 weeks after TAC in WT and Cand2 KO mice measured by echocardiography. (H) Fractional shortening 2 weeks after TAC in WT and Cand2 KO mice measured by echocardiography. (I) LV weight to body weight ratio of WT and Cand2 KO mice subjected to sham and TAC surgeries. (J) Nppa and Nppb mRNA levels 4 weeks after TAC measured by RT-qPCR. Error bars indicate means \pm SEM; ${ }^{*}-P \leq 0.05,{ }^{* *}-P \leq 0.01,{ }^{* * *}-P$ $\leq 0.0001$

\section{Figure 5. Cand2-dependent Grk5 expression.}

(A) Enrichment of Kyoto Encyclopedia of Genes and Genomes (KEGG) terms for differentially expressed genes in LV of Cand2 KO vs. WT mice (right panel). Volcano plot of up - (RNA-seq log2-fold change of count per million $>1$; red dots) and downregulated ( $>1$; blue dots) transcripts in LV after Cand2 KO (left plot). (B) Immunoblots and gel quantification of Grk5 protein levels in vitro after Cand2 KD with siRNA and Cand2 OE with AAV6. (C) Grk5 expression levels in NRCMs depleted of and overexpressing Cand2 measured by RT-qPCR. (D) Grk5 protein levels in vivo in LVs of WT and Cand2 KO mice 4 weeks after sham and TAC analyzed by immunoblotting (upper panel) and its quantification (left bottom graph), and Grk5 mRNA levels in indicated conditions (right bottom graph). (E) Representative immunoblot of Grk5 protein in cytoplasmic and nuclear fractions from left ventricle lysates of WT and Cand2 KO mice after TAC surgery. Lamin B - nuclear marker, Gapdh - cytoplasmic marker.

\section{Figure 6. Cand2 regulates MEF2 transcriptional activity.}

(A) Luciferase based quantification of MEF2 activity after Cand2 KD in NRCMs. (B) mRNA levels of MEF2-dependent genes Nr4a1 and Xirp2 in NRCMs with Cand2 KD. (C) mRNA levels of MEF2-dependent genes Nr4a1 and Xirp2 in NRCMs with Cand2 OE. (D) RT-qPCR analysis of MEF2 targets in LVs of WT and Cand2 KO mice after sham and TAC surgeries. (E) Grk5 protein, mRNA levels in NRCMs and MEF2-luciferase activity after 24h of Torin 1 treatment. (F) Cand2 and Grk5 protein levels and Rptor transcript level in NRCMs after Raptor KD analyzed by Western blot (G) Quantifications of Cand2 and Grk5 protein levels and Grk5 transcript level in NRCMs depleted of Raptor. (H), Nppa, and MEF2 targets Nr4a1 and Xirp2 mRNA levels in NRCMs after Raptor KD. Error bars indicate means \pm SEM; * $-P \leq 0.05$, ${ }^{* *}-P \leq 0.01,{ }^{* * *}-P \leq 0.001,{ }^{* * * *}-P \leq 0.0001$

\section{Figure 7. Cand2 and Cullin1 neddylation modulate Grk5 protein level.}

(A) Representative immunoblot and its quantification of protein levels of neddylated and unnedylated Cul1 in NRCMs after Cand2 OE. (B) Cul1 neddylation analyzed by Western blot in NRCMs depleted of Cand2. NRCMs were treated with MLN4924 to block Cul1 neddylation. The graphs show immunoblot quantification. (C) Cell size area measurement of NRCMs after Cand2 KD and neddylation inhibition (MLN). (D) Grk5 expression in NRCMs upon Cand2 KD and Cul1 neddylation inhibition examined by immunoblot and RT-qPCR. (E) The half-life of Grk5 in HeLa cells overexpressing Cand2 measured by cycloheximide chase. (F) Grk5 protein and mRNA levels in vitro after Cul1 KD and immunoblot quantification. (G) Comparison of NRCMs size with Cand2 KD, Grk OE after PE stimulation. Immunoblot confirms Cand $2 \mathrm{KD}$ and Grk5 OE. Error bars indicate means $\pm \mathrm{SEM}$; ${ }^{*}-P \leq 0.05$, ${ }^{*}$ - $P \leq 0.01$, ${ }^{* * *}-P \leq 0.001,{ }^{* * * *}-P \leq 0.0001(\mathrm{H})$ Model of Cand2-dependent stabilization of Grk5 by Cul1 neddylation inhibition in mTOR-dependent manner. 


\section{Expanded View Figures}

\section{EV 1. Specific inhibition of mTOR pathway by Torin1.}

(A) NRCMs treatm with increasing concentration of Torin1 for 24h analyzed by immunoblotting. mTORC1 inhibition was detected by total and phosphorylated levels of S6RP and 4E-BP1. mTOR2 activity was analyzed by Akt phosphorylation. PI3K pathway was analyzed by the phosphorylation level of its downstream target Erk1/2. (B) Expression of mTOR-dependent transcripts. eEF2 and Rps5 levels were analyzed by immunoblot (left panel) and RT-qPCR (right panel) after PE and Torin1 treatment. Csq - calsequestrin has been used as a housekeeping protein. (C) mTOR-dependent expression of hypertrophy markers. RT-qPCR analysis of Nppa and Nppb mRNA levels after PE and Torin 1 treatment of NRCMs. (D) Polysome profiles of control and PE- and Torn1-treated NRCMs.

\section{EV 2}

(A) Representative bright-field images of isolated cardiac myocytes stimulated with (PE, 50 $\mu \mathrm{M}$ for $24 \mathrm{~h}$ ) with or without mTOR inhibition (Torin 1,150 nM) and quantification of cell surface area measurements from control, PE treatment, and Torin 1 treatment. $n=4$ independent experiments, ${ }^{*}-P<0.01$ different from PE. Scale bar $100 \mu \mathrm{m}$ (B) Luciferase activity from control, $\mathrm{PE}$ treated, and Torin 1 treated cardiac myocytes. $\mathrm{n}=2$ independent experiments, * $P$ $<0.01$ different from PE. (C) Heart weight (HW) to body weight (BW) ratio (HW/BW) in control and Torin 1 -treated mice $(10 \mathrm{mg} / \mathrm{kg} \mathrm{BW}) 2$ days after sham or TAC surgery. ${ }^{*}-P<0.05$ different from TAC. $n=3$ per group

\section{EV 3. Cand2 knock-out mouse model characterization.}

(A) Cand2 protein levels in WT, heterozygous, and homozygous Cand2 knock-out mice analyzed by immunoblotting in muscle tissues. (B) The percentage of ejection fraction in WT and Cand2 KO analyzed by echocardiography prior sham/TAC surgeries. (C) The baseline of LV weight (mg) to body weight (g) ratios of WT and KO Cand2 mice. (D) RT-qPCR analysis of Cand2 and Cand1 transcript levels in WT and Cand2 KO mice. (E) Nppa and Nppb (F) transcripts in WT and Cand2 KO mice.

\section{EV 4. Cand2 interacts with Cul1 and Grk5 in cardiomyocytes.}

(A) Cand2, Cul1, and Grk5 association analyzed by co-immunoprecipitation (IP) of endogenous Grk5 with Cand2-specific antibody and immunoblotting ( $\mathrm{i}$ - input; IgG - negative control, IP with anti-rabbit IgG antibody) and Immunoprecipitation of endogenous Cul4 with Cand2 specific antibody ( $\mathrm{i}$ - input; $\lg G$ - negative control, IP with anti-rabbit lgG antibody). (B) Interaction of Cand2 with Grk5 measured by proximity ligation assay (PLA). PLA (red) in primary neonatal rat (NRCMs) and cardiomyocytes isolated from hearts of wild-type (C) and Cand2 KO (D) neonatal mice (NMCMs). Each red dot represents the interaction of endogenous Cand2 and Grk5. No dots were detected in mouse cardiomyocytes from Cand2 KO hearts. (E) PLA of Cand2 and Cullin1 in NRCMs and (F) NMCMs from WT and (G) Cand2 KO mice. Red dots represent the Cand2 and Cul1 association. No dots were detected in mouse cardiomyocytes from Cand2 KO hearts. Scale bar $20 \mu \mathrm{m}$

\section{EV 5. Cand2 regulates Grk5 protein level by Cullin1 neddylation inhibition.}

(A) RT-qPCR analysis of Cand2 mRNA levels in NRCMs after neddylation inhibition (MLN) and Cand2 KD with siRNA. (B) Cul1 expression in NRCMs overexpressing (left graph) and depleted of Cand2 (right graph) examined by RT-qPCR. (C) Grk5 and Cul1 mRNA levels in NRCMs overexpressing Grk5 measured by RT-qPCR. 
Figure 1

A

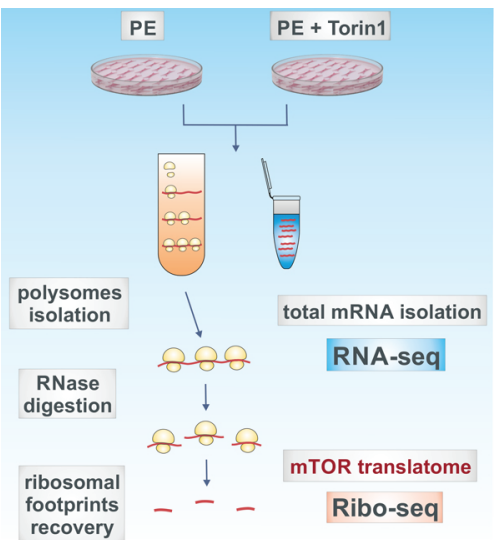

B

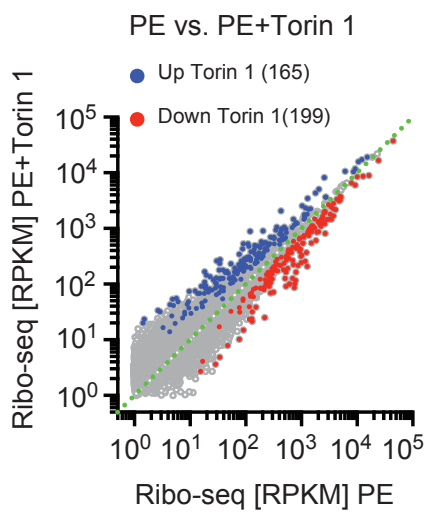

C

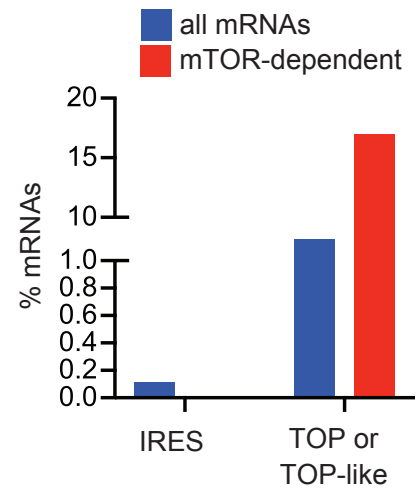

D

Torin1-responsive

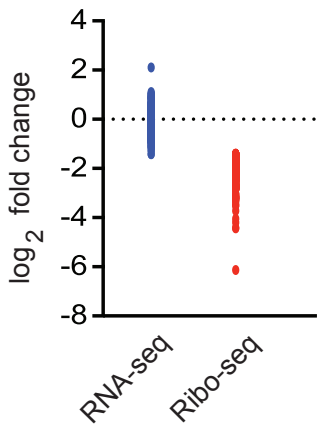

$\mathbf{F}$

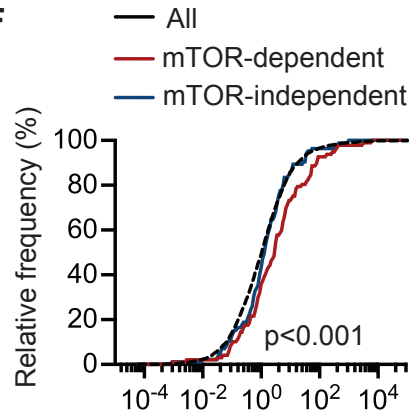

Ribo-seq TAC $2 d$ vs. Sham $\log _{2}$ fold change

G response to

regulation of apoptotic process

purine nucleoside cellular protein
metabolic process response to organonitrogen triphosphate regulation of cell death cellular respon
med Gell
substance regulation of programmed cel ribonucleoside triphosphate nucleoside triphosphate
metabofic process modification-dependent protein catabolic macromolecule catabolic process process

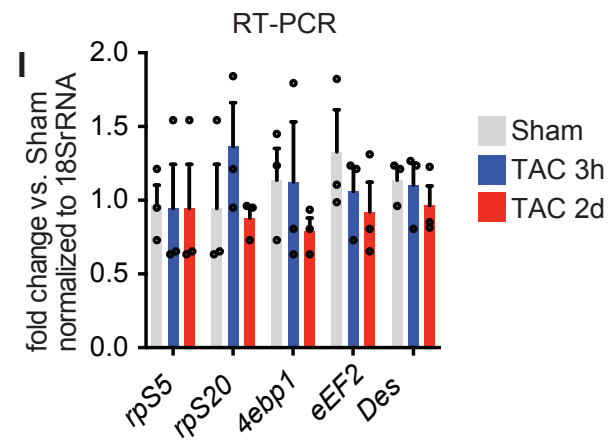

\section{- All}

- mTOR-dependent

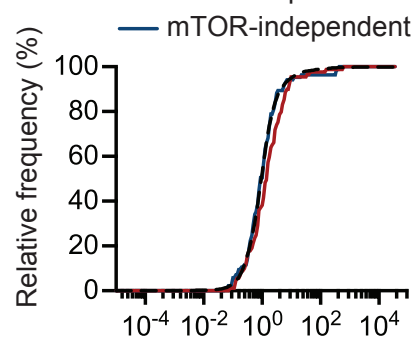

RNA-seq TAC 2d vs. Sham $\log _{2}$ fold change
rpS5

Csq

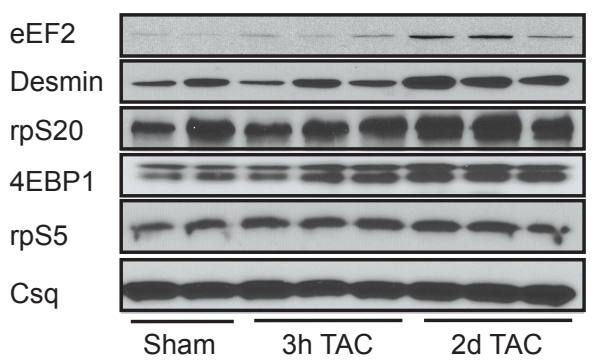


Figure 3

A

\section{Cand2} Gapdh

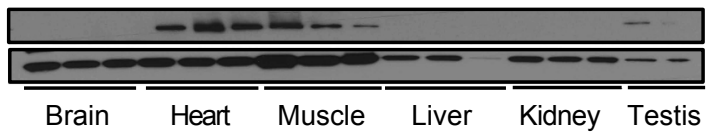

$-1.5\urcorner \quad$ Cand2
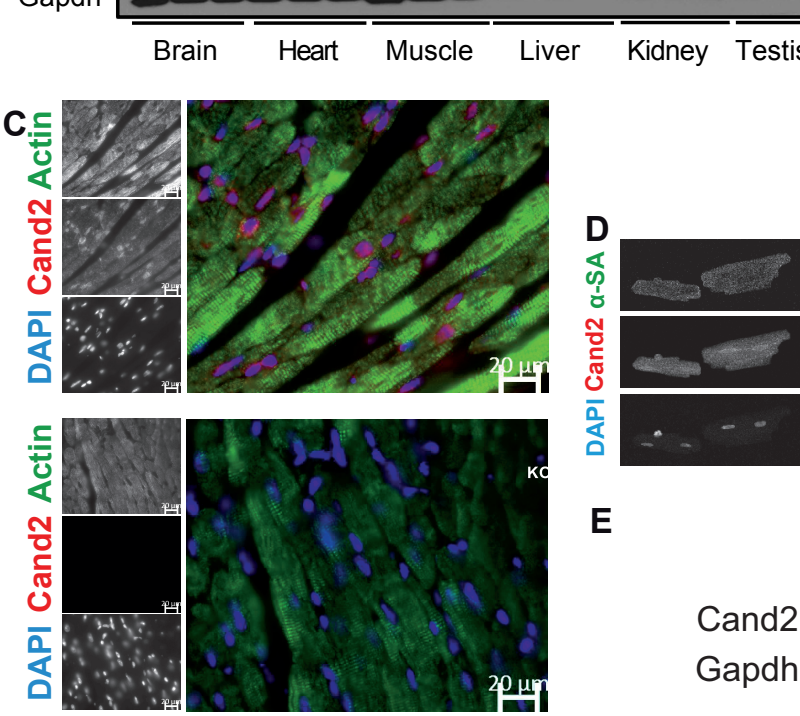

E Histone 3D

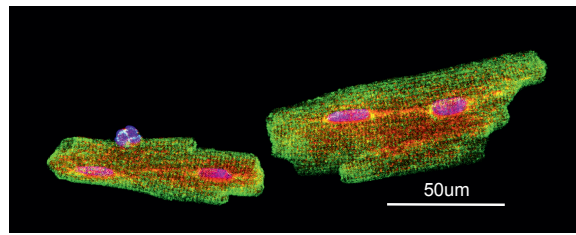

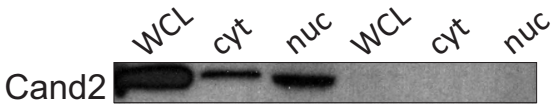

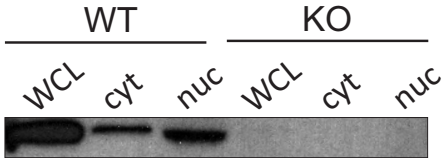
Gapdh

B
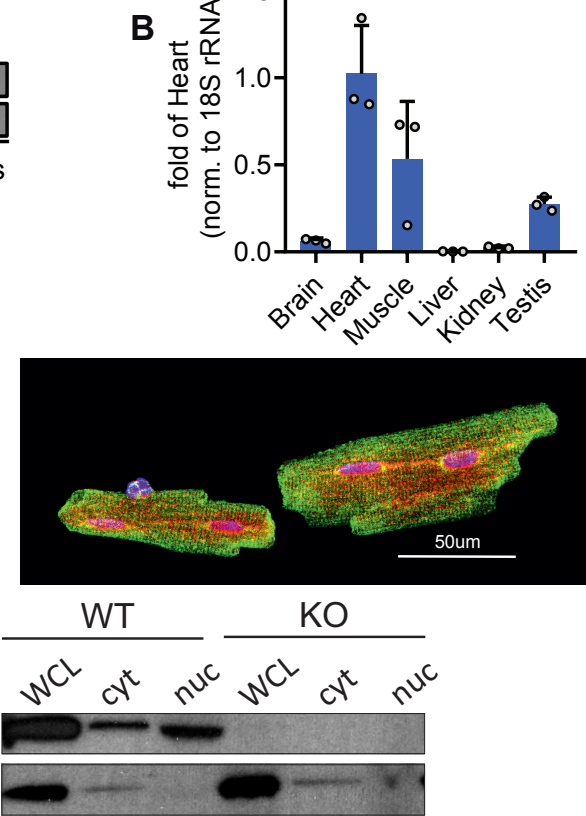

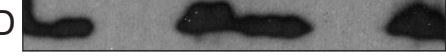


Figure 4

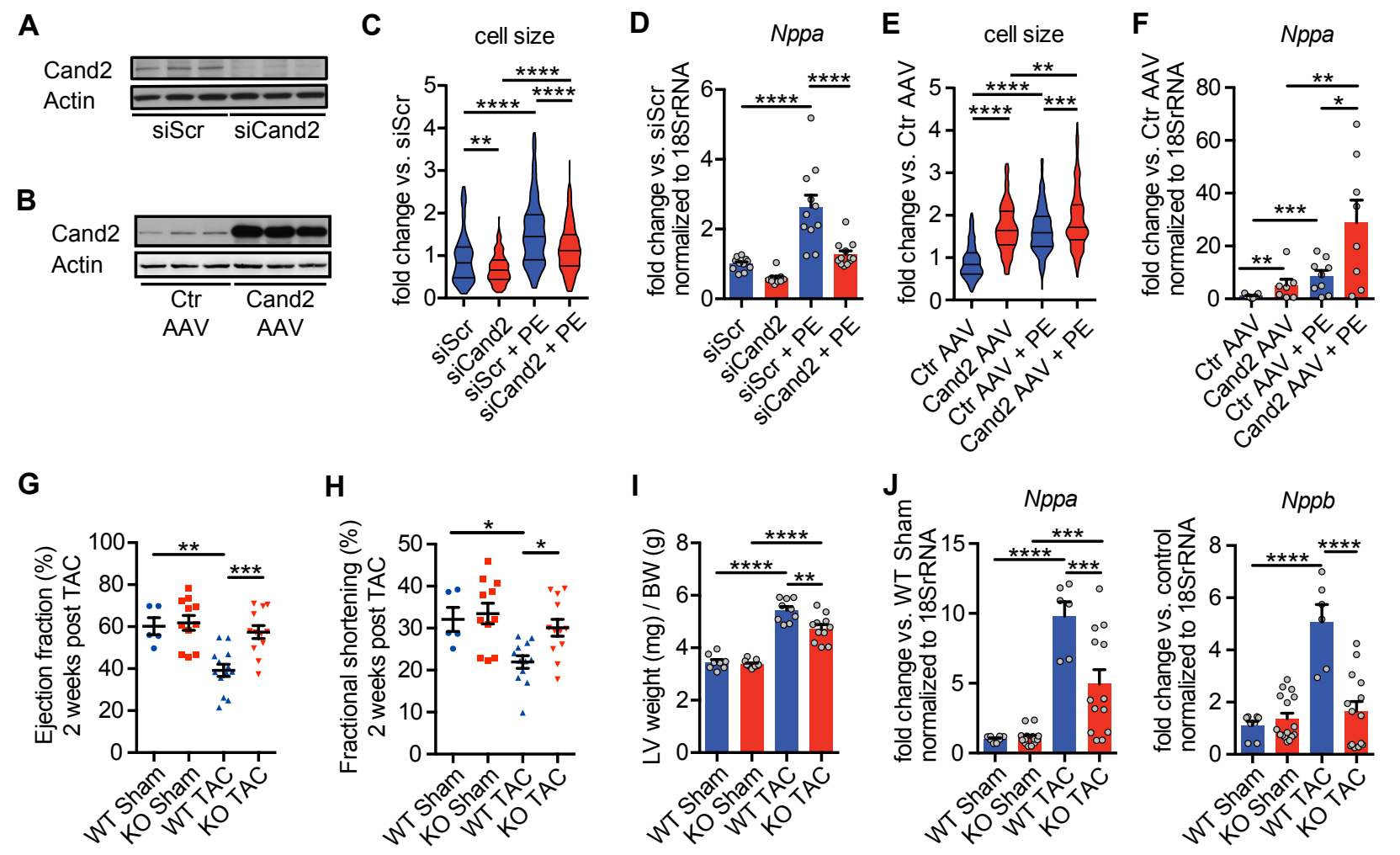




\section{Figure 5}

A
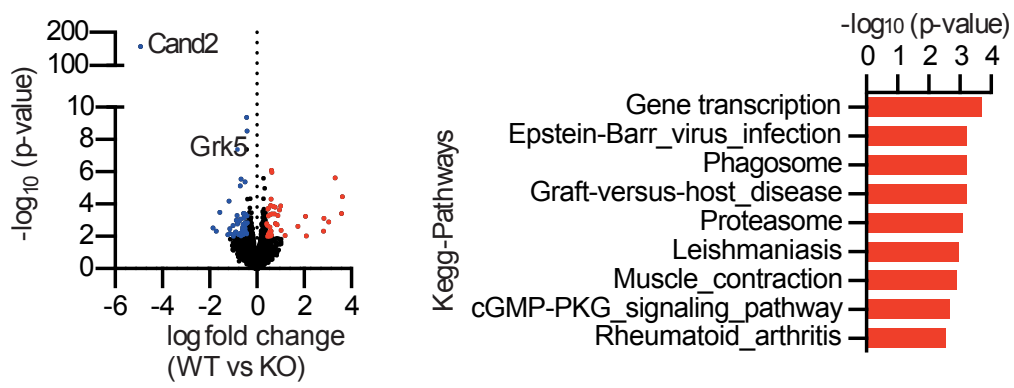

D
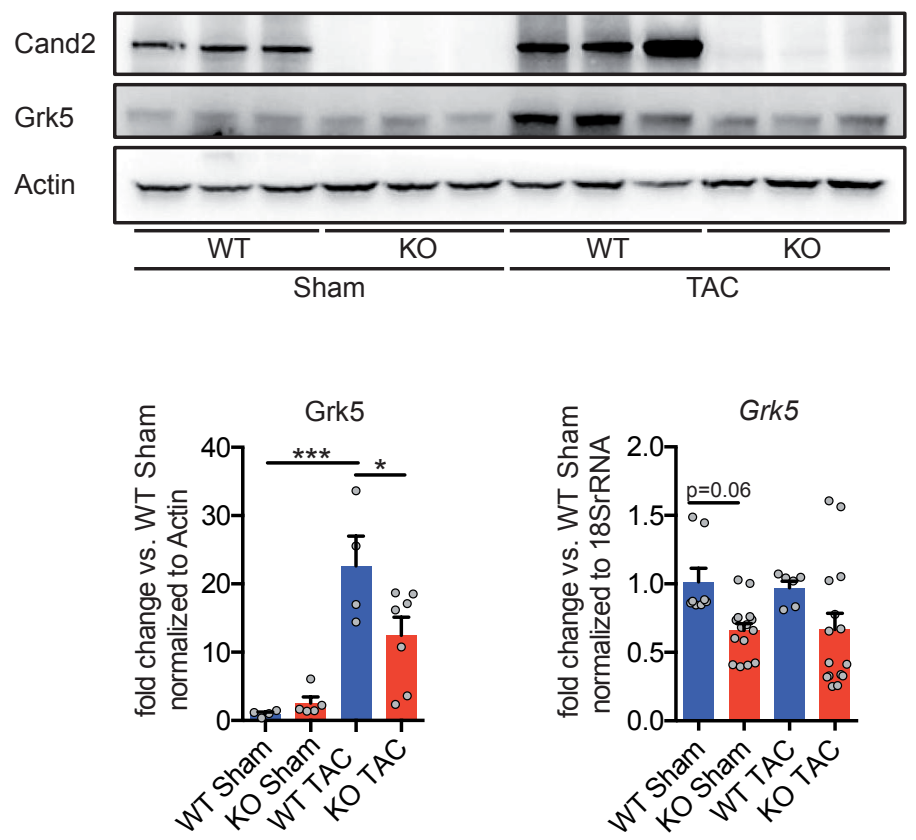

B Cand2 -

Grk5 $-\cdots$

Gapdh

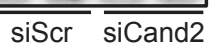

Cand2 $\square-\cdots$

Grk5 --

Gapdh

Ctr AAV Cand2

AAV

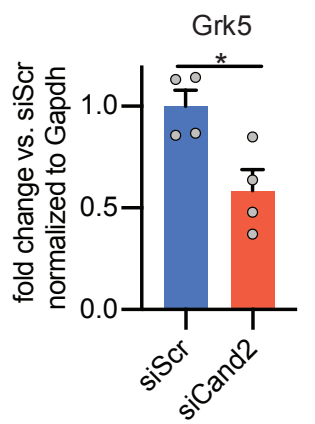

C
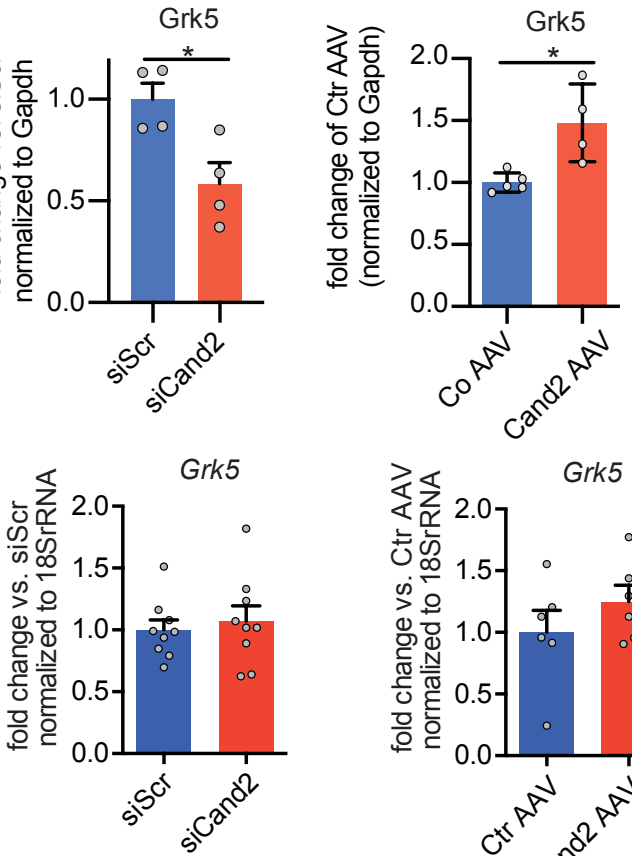

E
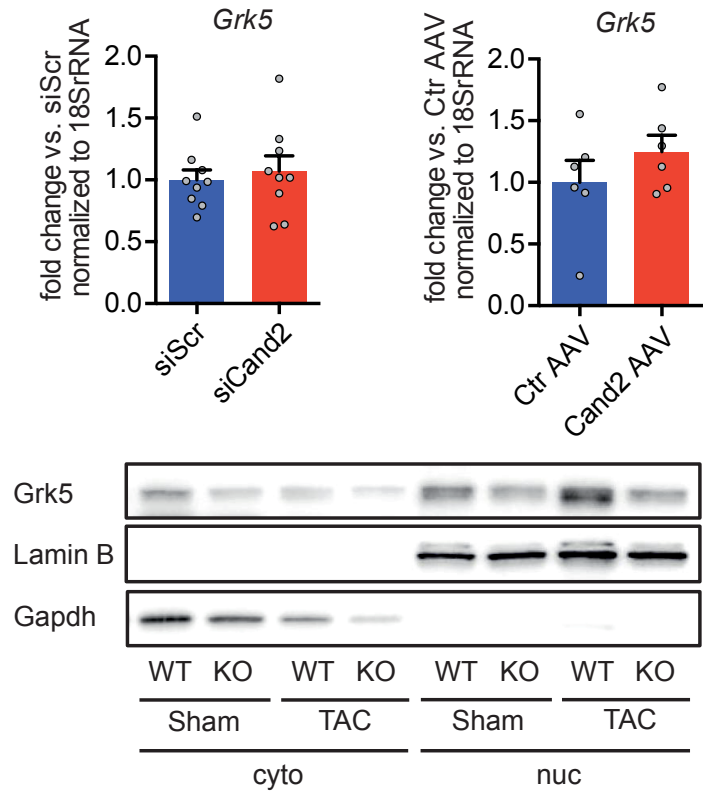


\section{Figure 6}

A

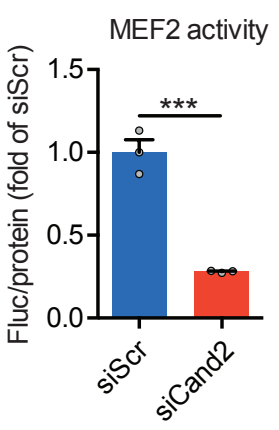

E

Grk5 pRibS6 Actin
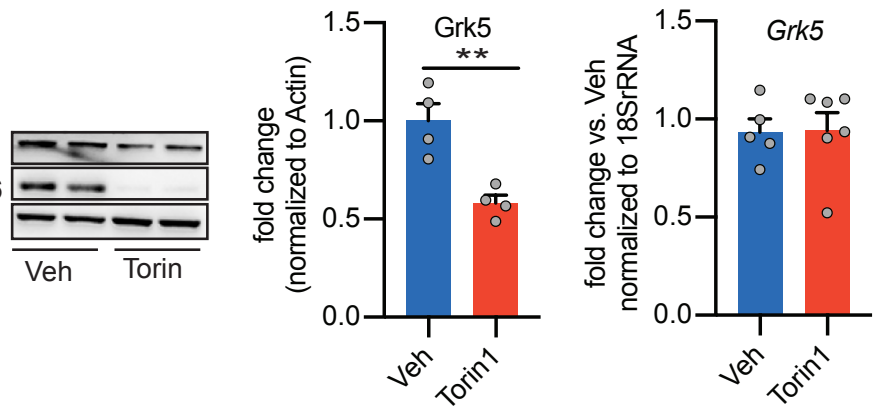

G

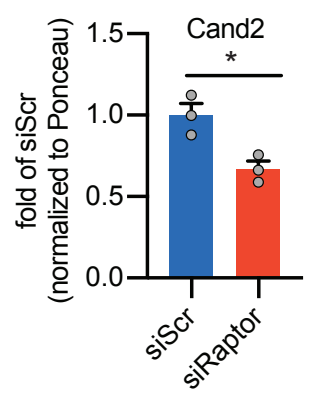

Nr4a1
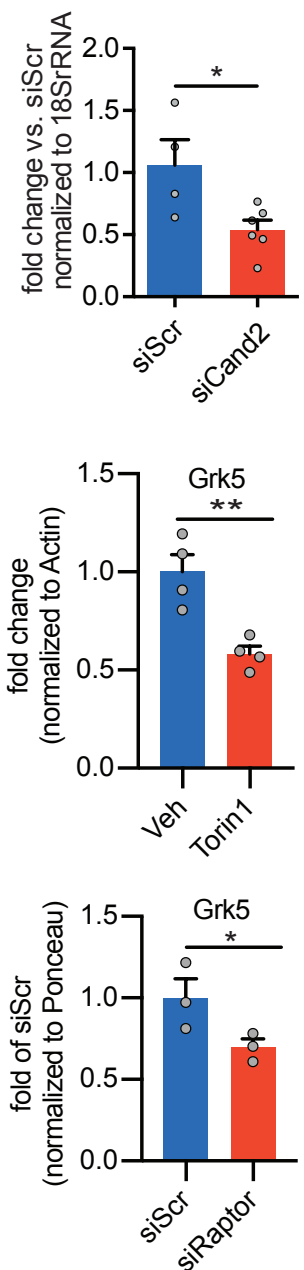
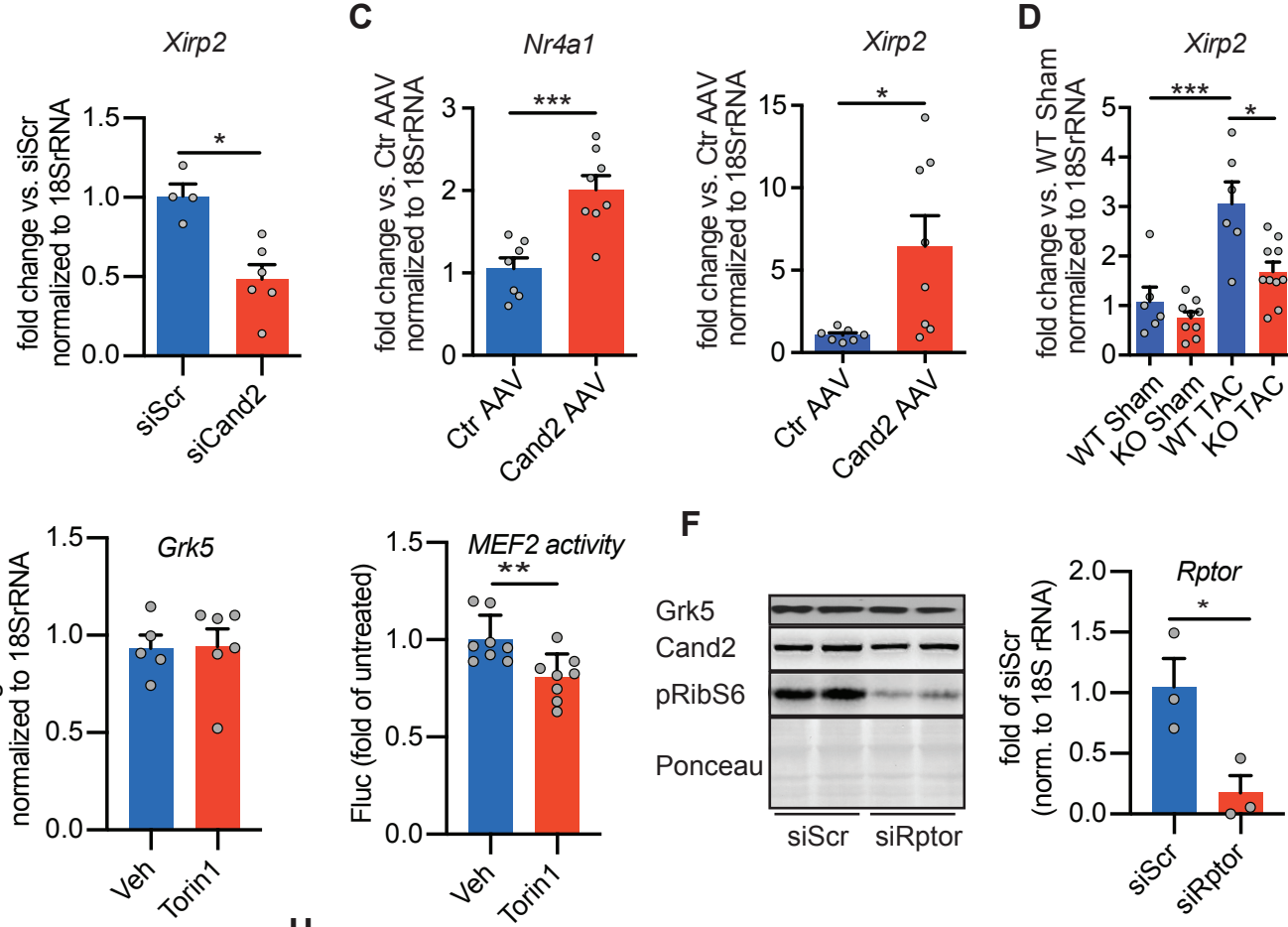

H

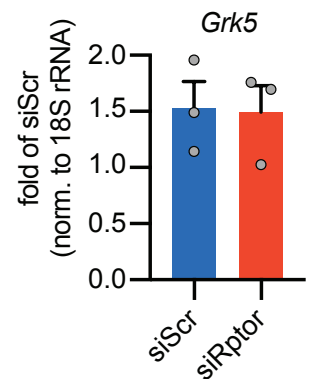

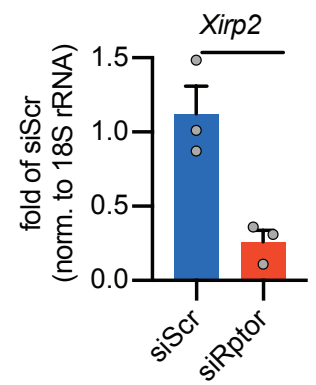
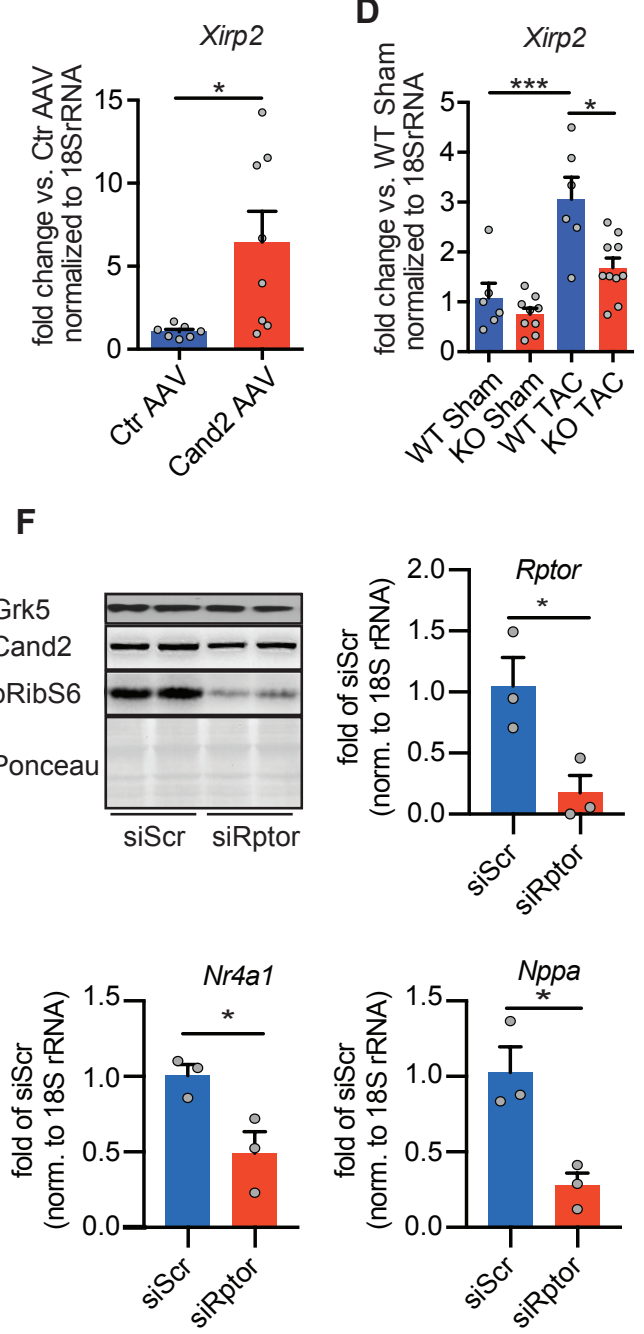
Figure 7

A Cand2

Cul1

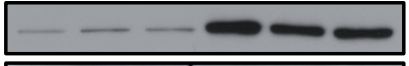

Ponceau

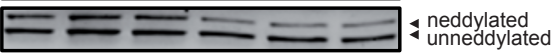

Ctr AAV Cand2 AAV

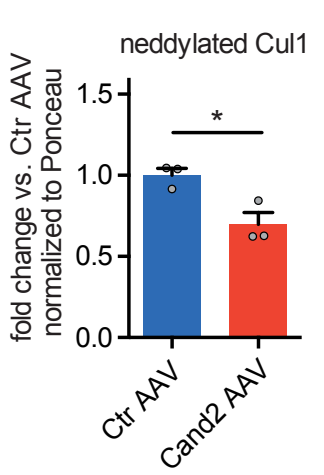

unnedylated Cul1

D
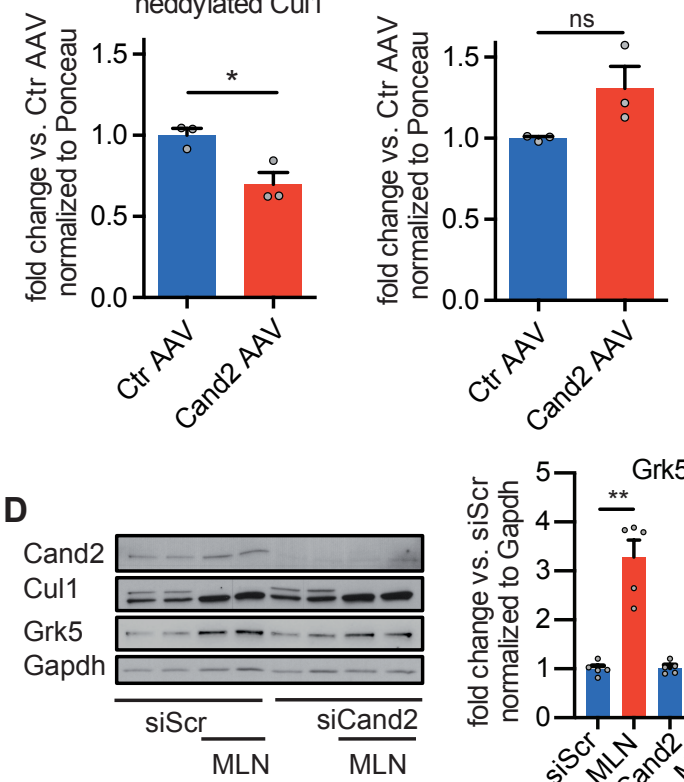

F

Cul1

Grk5

Actin

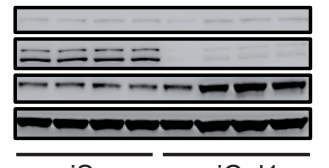

siScr siCul1

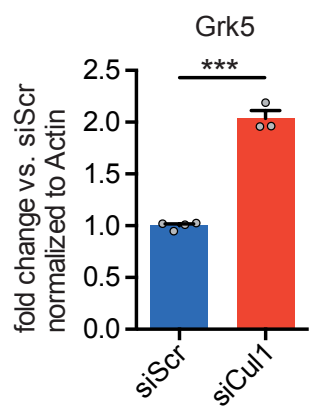

B

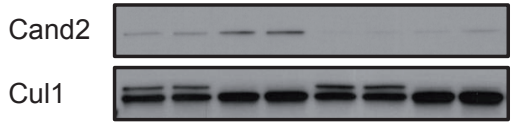

Actin

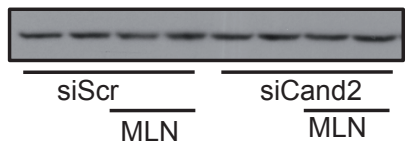

neddylated Cul1

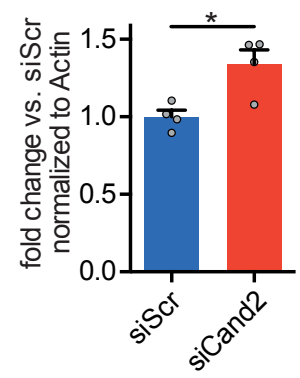

unneddylated Cul1
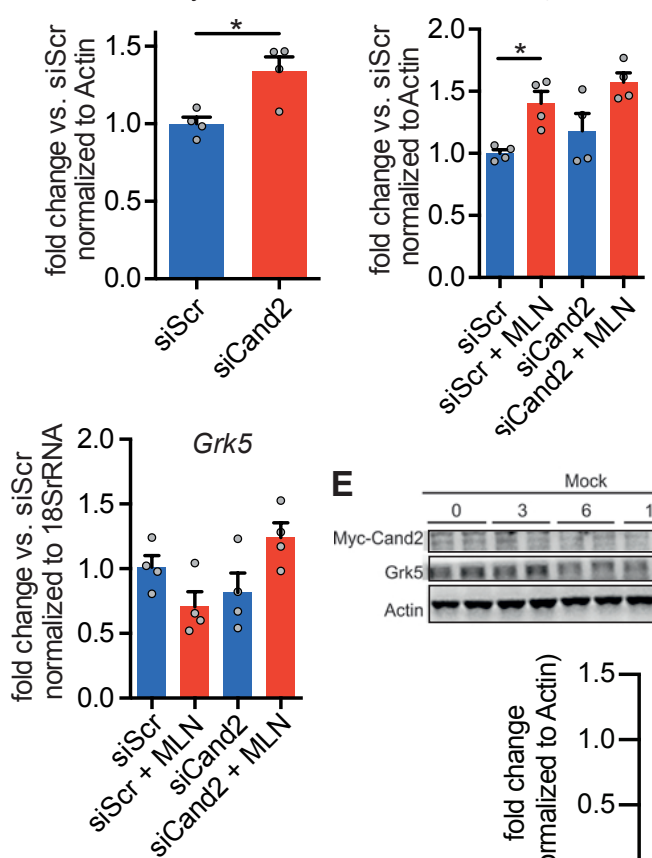

Grk5

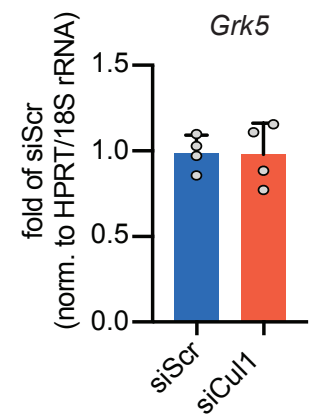

Cand2

Cul1

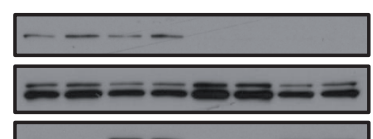

Grk5

Gapdh

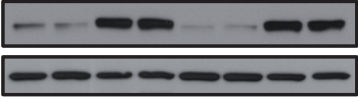

$\frac{\text { siScr }}{\mathrm{Ctr}} \frac{\mathrm{siCand}}{\mathrm{Grk} 5} \frac{\mathrm{str}}{\mathrm{Ctr}} \frac{\mathrm{Grk} 5}{\mathrm{G}}$ cell size

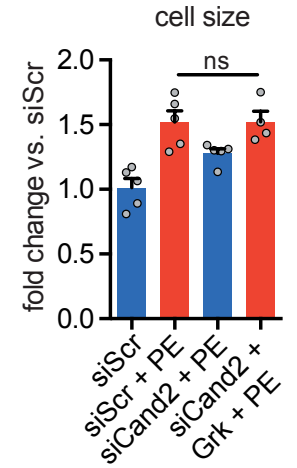

cell size

C

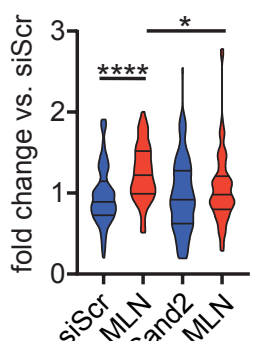

के
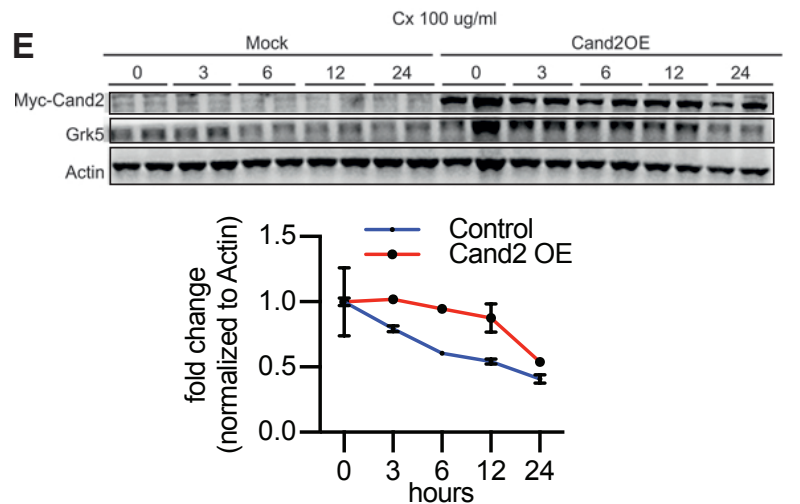

$\mathrm{H}$

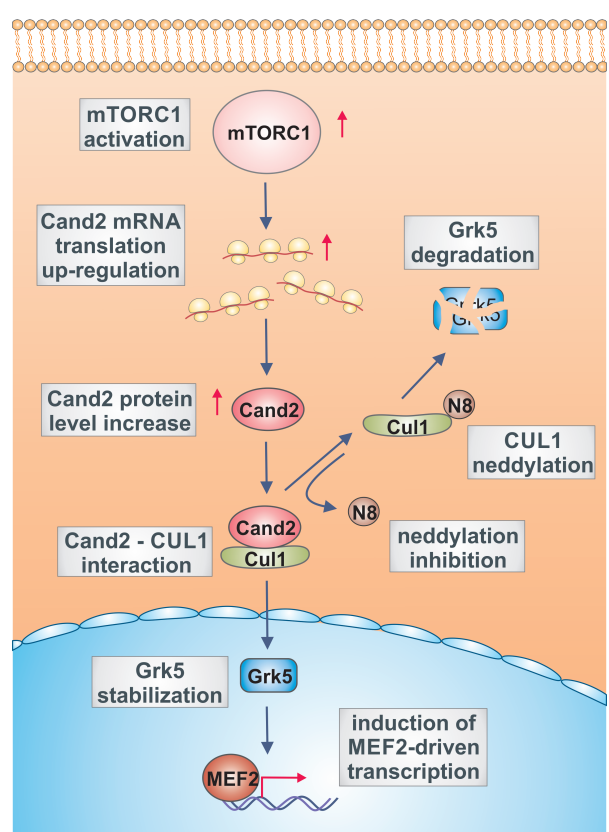

